Central Washington University

ScholarWorks@CWU

All Master's Theses

Master's Theses

Spring 2018

\title{
Decreasing Occlusion and Increasing Explanation in Interactive Visual Knowledge Discovery
}

\author{
Abdulrahman Ahmed Gharawi \\ Central Washington University, gharawia@cwu.edu
}

Follow this and additional works at: https://digitalcommons.cwu.edu/etd

Part of the Artificial Intelligence and Robotics Commons, and the Graphics and Human Computer Interfaces Commons

\section{Recommended Citation}

Gharawi, Abdulrahman Ahmed, "Decreasing Occlusion and Increasing Explanation in Interactive Visual Knowledge Discovery" (2018). All Master's Theses. 941.

https://digitalcommons.cwu.edu/etd/941

This Thesis is brought to you for free and open access by the Master's Theses at ScholarWorks@CWU. It has been accepted for inclusion in All Master's Theses by an authorized administrator of ScholarWorks@CWU. For more information, please contact scholarworks@cwu.edu. 


\title{
DECREASING OCCLUSION AND INCREASING EXPLANATION IN INTERACTIVE VISUAL KNOWLEDGE DISCOVERY
}

\author{
A Thesis \\ Presented to \\ The Graduate Faculty \\ Central Washington University
}

In Partial Fulfillment

of the Requirements for the Degree

Master of Science

Computational Science

by

Abdulrahman Ahmed Gharawi

May 2018 


\section{CENTRAL WASHINGTON UNIVERSITY}

Graduate Studies

We hereby approve the thesis of

Abdulrahman Ahmed Gharawi

Candidate for the degree of Master of Science

APPROVED FOR THE GRADUATE FACULTY

Dr. Boris Kovalerchuk, Committee Chair

Dr. Razvan Andonie

Dr. Szilard Vajda

Dean of Graduate Studies 


\begin{abstract}
DECREASING OCCLUSION AND INCREASING EXPLANATION IN INTERACTIVE VISUAL KNOWLEDGE DISCOVERY
\end{abstract}

by

Abdulrahman Ahmed Gharawi

May 2018

Lack of explanation and occlusion are the major problems for interactive visual knowledge discovery, machine learning and data mining in multidimensional data. This thesis proposes a hybrid method that combines visual and analytical means to deal with these problems. This method, denoted as FSP, uses visualization of n-D data in 2-D in a set of Shifted Paired Coordinates (SPC). SPC for $n-D$ data consists of $n / 2$ pairs of Cartesian coordinates that are shifted relative to each other to avoid their overlap. Each n-D point is represented as a directed graph in SPC. It is shown that the FSP method simplifies pattern discovery in n-D data providing explainable rules in a visual form with significantly decrease of the cognitive load for analysis of $n-D$ data. The computational experiments on real data has shown its efficiency on both training and validation data. 


\section{ACKNOWLEDGEMENTS}

Firstly, I would like to express my sincere gratitude to my advisor Profs. Boris Kovalerchuk for his continuous support and endless advice, for his patience, motivation, and immense knowledge. His patient guidance, encouragement, and advice helped me all during the time of my research and master's study.

Besides my advisor, I would like to thank the rest of my thesis committee: Profs. Razvan Andonie and Szilárd Vajda, for their insightful comments, endless advice, and encouragement.

My sincere thanks also goes to professor Donald Davendra who provided me with unfailing support and continuous encouragement throughout my years of study.

Last but not least, I would like to thank my family: my parents, my wife Jumana Alsubhi, and my daughter, for supporting me spiritually throughout writing this thesis and my life in general. 
TABLE OF CONTENTS

Chapter

Page

I INTRODUCTION ....................................................................... 1

SHIFTED PAIRED COORDINATES .......................................... 2

II VISUAL KNOWLEDGE DISCOVERY ............................................... 5

FSP ALGORITHM ................................................................. 5

FREQUENCY VISUALIZATION ALGORITHM ............................ 10

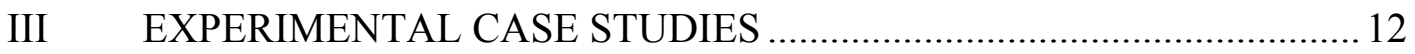

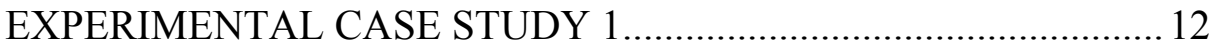

EXPERIMENTAL CASE STUDY 2 ….......................................... 18

EXPERIMENTAL CASE STUDY 3 ............................................. 23

EXPERIMENTAL CASE STUDY 4 ............................................ 27

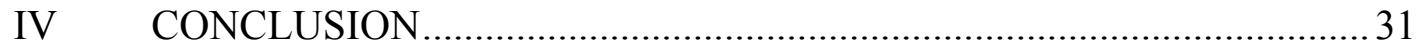

COMPARISON WITH PUBLISHED RESULTS ............................. 31

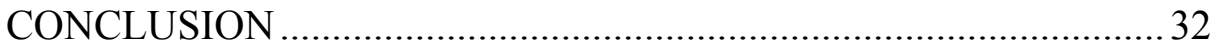

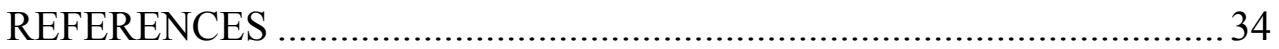

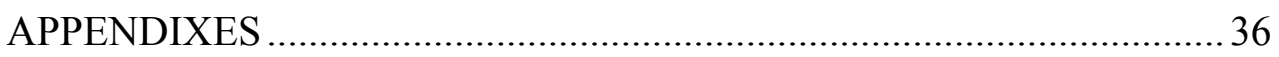

Appendix A-WISCONSIN BREAST CANCER (WBC).................. 36

Appendix B-IONOSPHERE DATASET ...................................... 36

Appendix C-ABALONE DATASET .............................................. 36

Appendix D-MNIST DATASET …............................................. 37 


\section{LIST OF TABLES}

Table

Page

1 Number of cases that satisfy the if part of Rule 1 in 11 random 70\%:30\%

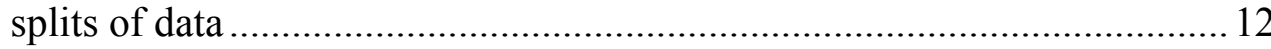

2 Precision, recall and coverage of Rule 1 in 11 random 70\%:30\% splits

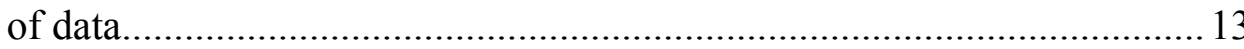

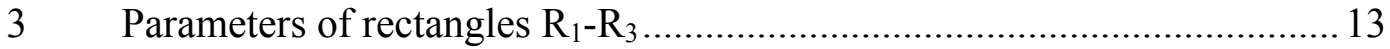

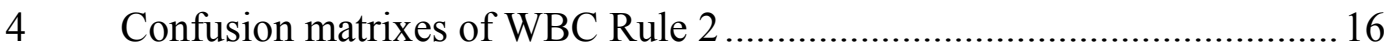

5 Parameters of rectangles $\mathrm{R}_{4}-\mathrm{R}_{6}$ in normalized coordinates .................... 17

6 Confusion matrix of combined rules 1 and 3 on all 688 cases .................. 17

$7 \quad$ Number of cases that satisfy the Ionosphere Rule 1 in 11 random 70\%:30\% splits of data ................................................................. 19

8 Precision, recall and coverage of Ionosphere Rule 1 in 11 random 70\%:30\% splits of data

9 Parameters of rectangles $\mathrm{R}_{1}-\mathrm{R}_{10}$ in normalized coordinates .................... 19

10 Confusion matrixes of the Ionosphere Rule 2.................................. 21

11 Number of cases satisfying the if part of Abalone Rule 1 in 11 random

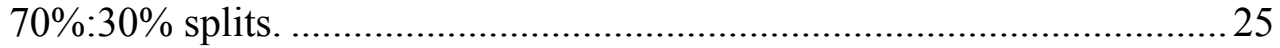

12 Precision, recall and coverage of Rule 1 in 11 random 70\%:30\%

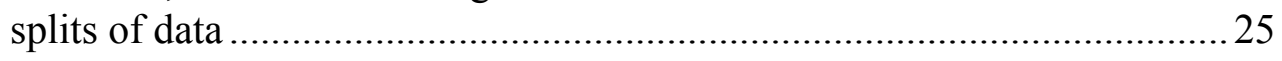

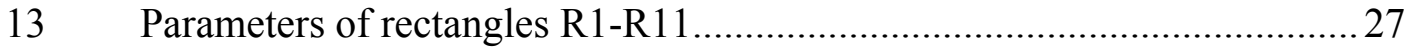

14 Confusion matrixes of Abalone Rule 2 2................................................ 27 


\section{LIST OF FIGURES}

Figure

$1 \quad 6$-D point $\mathrm{a}=(3,2,1,4,2,6)$ in Shifted Paired Coordinates

2 A set of 688 Wisconsin Breast Cancer (WBC) data visualized in SPC as 2-D graphs of 10-D points with benign cases in Red and malignant cases in Blue.

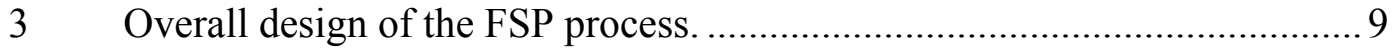

$4 \quad$ Alternative frequency visualization ................................................ 11

$5 \quad$ WBC data in SPC as graphs representing 10-D points that go through $\mathrm{R}_{1}$ without showing frequency of cases. Magenta boxes show rectangles $\mathrm{R}_{1}-\mathrm{R}_{3}$

6 WBC data in SPC as graphs representing 10-D points that satisfy Rule 1 (go through $\mathrm{R}_{1}$ and not coming to $\mathrm{R}_{2}$ and $\mathrm{R}_{3}$ shown in magenta.

Wide Redlines show the frequency of Red cases

7 Remaining WBC cases not covered by Rule (dominated by Blue class) ... 15

8 WBC data in 4-D SPC as graphs in coordinates $\left(\mathrm{X}_{9}, \mathrm{X}_{8}\right)$ and $\left(\mathrm{X}_{6}, \mathrm{X}_{7}\right)$ that are used by Rule 1 , i.e., WBC cases that go through $\mathrm{R}_{1}$ and not go to $\mathrm{R}_{2}$ and $\mathrm{R}_{3}$ in these coordinates

9 The precision, recall and total coverage of combined Rule 1 and Rule 3 .. 17

10351 Ionosphere cases in the SPCs as graphs of 34-D points (good cases in Red and bad cases in Blue). Rectangles that are used in Ionosphere Rule 1 are in magenta......

11 34-D Ionosphere cases covered by Ionosphere Rule 1 Rectangles from Rule 1 are in magenta

12 Remaining Ionosphere cases (cases not covered by Ionosphere Rule 1) ... 22

13 Ionosphere cases in 20-D points covered by the Ionosphere Rule 1 22 


\section{LIST OF FIGURES}

Figure

14 Ionosphere cases in 20-D points covered by Rule 1

15 Ionosphere cases in 20-D points not covered by Rule 1.

16 A set of 2870 Abalone data visualized in the SPCs, as graphs of 8-D points, with the male cases in Red, and the infant cases in Blue. Rectangles used in Abalone Rule 1 are in magenta.

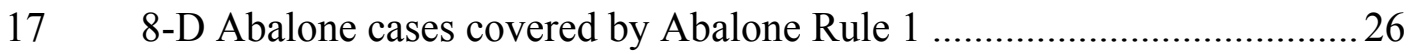

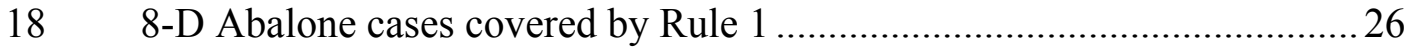

19 Remaining Abalone cases (cases not covered by Abalone Rule 1) show the frequency of Red cases ................................................................. 27

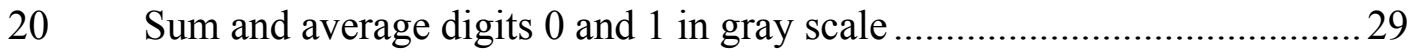

21 SPC pairs presented as black dots in $22 \times 22$ empty image for 1 and 0 ......29

22 SPC pairs presented as dark dots in the average of sum image of 1 and 0 30

23 SPC pairs presented as dark dots in the average of sum image of 1 and 0 magenta..... 


\section{CHAPTER I}

\section{INTRODUCTION}

For a long time, lack of explanation and occlusion have been the major problems for interactive visual knowledge discovery, data mining and machine learning in multidimensional data. This thesis proposes a hybrid method that combines visual and analytical means to deal with these problems in visual knowledge discovery. The proposed method, denoted as FSP, uses visualization of $n-D$ data in 2-D in a type of General Line Coordinates (GLC) [Kovalerchuk, Grishin, 2017, Kovalerchuk, 2018] known as Shifted Paired Coordinates (SPC). A set of Shifted Paired Coordinates for $n-D$ data consists of $n / 2$ pairs of Cartesian coordinates that are shifted relative to each other without overlap. Each n-D point $A$ is represented as a directed graph $A^{*}$ in SPC, where each node of the graph is a 2-D projection of $A$ in a respective pair of the Cartesian coordinates.

The proposed FSP method significantly decreases cognitive load for analysis of n-D data and simplifies discovery of explainable patterns in n-D data. At the upper level, the steps of the FSP are: (1) Filtering out less efficient visualizations from multiple SPC visualizations, (2) Searching for sequences of paired coordinates that are more efficient, and (3) Presenting the SPC visualizations only with better sequences to the analyst. FSP includes the randomized search for pairs of coordinates and explainable "rectangular" classification rules with maximized accuracy on training and validation data.

The computational experiments with the 9-D Wisconsin Breast Cancer data, 33-D Ionosphere data, and 8-D Abalone data from UCI Machine Learning repository show efficiency of the FSP method on training and validation data. The visualization process in SPC is reversible, i.e., all n-D information is visualized and can be restored from visualization for each 
n-D case. This hybrid visual analytics method allows classifying data in a way that can be communicated to the domain experts such as medical doctors in the explainable/understandable and visual form.

\section{Shifted Paired Coordinates: Challenge and Opportunity to Better Visualization}

The Shifted Paired Coordinates (SPC) visualization of n-D data requires splitting $n$ coordinates $\mathrm{X}_{1}-\mathrm{X}_{n}$ to pairs producing $n / 2$ non-overlapping pairs $\left(\mathrm{X}_{i}, \mathrm{X}_{j}\right)$, such as $\left(\mathrm{X}_{1}, \mathrm{X}_{2}\right),\left(\mathrm{X}_{3}, \mathrm{X}_{4}\right)$, $\left(\mathrm{X}_{5}, \mathrm{X}_{6}\right), \ldots,\left(\mathrm{X}_{n-1}, \mathrm{X}_{n}\right)$ [Kovalerchuk, 2014; Kovalerchuk, Grishin, 2017, Kovalerchuk, 2018]. In SPC, each pair $\left(\mathrm{X}_{i}, \mathrm{X}_{j}\right)$ is represented as a separate orthogonal Cartesian Coordinates $(\mathrm{X}, \mathrm{Y})$, where $\mathrm{X}_{i}$ is $\mathrm{X}$ and $\mathrm{X}_{j}$ is $\mathrm{Y}$.

In SPC visualization design each coordinate pair $\left(\mathrm{X}_{i}, \mathrm{X}_{j}\right)$ is shifted relative to other pairs to avoid their overlap. This creates $n / 2$ scatter plots. Next in SPC, for each n-D point $\mathbf{x}=\left(x_{1}, x_{2}, \ldots, x_{n}\right)$, the point $\left(x_{1}, x_{2}\right)$ in $\left(\mathrm{X}_{1}, \mathrm{X}_{2}\right)$ is connected to the point $\left(x_{3}, x_{4}\right)$ in $\left(\mathrm{X}_{3}, \mathrm{X}_{4}\right)$ and so on until point $\left(x_{n-2}, x_{n-1}\right)$ in $\left(\mathrm{X}_{n-2}, \mathrm{X}_{n-1}\right)$ is connected to the point $\left(x_{n-1}, \mathrm{X}_{n}\right)$ in $\left(\mathrm{X}_{n-1}, \mathrm{X}_{n}\right)$ to form a directed graph $\mathbf{x}^{*}$. Figure 1 shows the same data visualized in SPC in two different ways due to different pairing of coordinates.

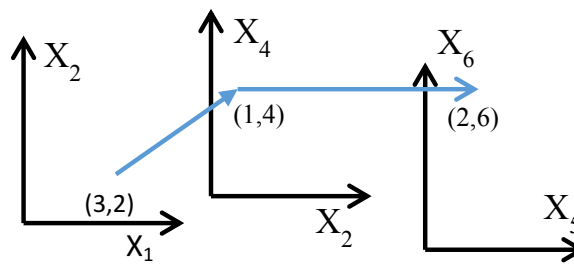

Figure a: Point a in $\left(\mathrm{X}_{1}, \mathrm{X}_{2}\right),\left(\mathrm{X}_{3}, \mathrm{X}_{4}\right),\left(\mathrm{X}_{5}, \mathrm{X}_{6}\right)$ as a sequence of pairs $(3,2),(1,4)$ and $(2,6)$.

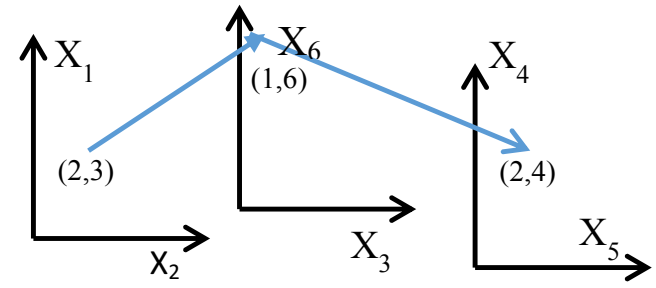

Figure b: Point a in $\left(\mathrm{X}_{2}, \mathrm{X}_{1}\right),\left(\mathrm{X}_{3}, \mathrm{X}_{6}\right),\left(\mathrm{X}_{5}, \mathrm{X}_{4}\right)$ as a sequence of pairs $(2,3),(1,6)$ and $(2,4)$.

Figure 1: 6 -D point $\mathrm{a}=(3,2,1,4,2,6)$ in Shifted Paired Coordinates. 
In general, there are multiple combinatorial ways to form pairs of coordinates for SPC and to sequence pairs. The SPC visualization graphs $\mathbf{x}_{k}{ }^{*}$ of each given n-D point $\mathbf{x}$ differ for different sequences $S_{k}$ of pairs of coordinates. Fig. 1a illustrates it for a 6-D point $\mathbf{a}=(3,2,1,4,2,6)$ visualized in pairs $\left(\mathrm{X}_{1}, \mathrm{X}_{2}\right),\left(\mathrm{X}_{3}, \mathrm{X}_{4}\right),\left(\mathrm{X}_{5}, \mathrm{X}_{6}\right)$, and Fig. $1 \mathrm{~b}$ shows this point visualized in pairs $\left(\mathrm{X}_{2}, \mathrm{X}_{1}\right),\left(\mathrm{X}_{3}, \mathrm{X}_{6}\right),\left(\mathrm{X}_{5}, \mathrm{X}_{4}\right)$

The SPC allows visualizing each individual n-D point losslessly, but together graphs of multiple n-D points occlude each other. See Fig. 2. This creates a difficulty for discovering patterns to classify cases of opposing classes in SPC. In Fig. 2 some areas are visibly dominated by cases of the specific color. However, it is not sufficient to build discrimination rules to classify cases visually. It required an addition analytical process. Such process is proposed in this thesis. SPC visualizations with some sequences $S_{k}$ can reveal classification patterns of n-D data better than with using other sequences. The dependence of the visualization from the different pairing coordinates creates a challenge and an opportunity to find better pairs and their sequences.

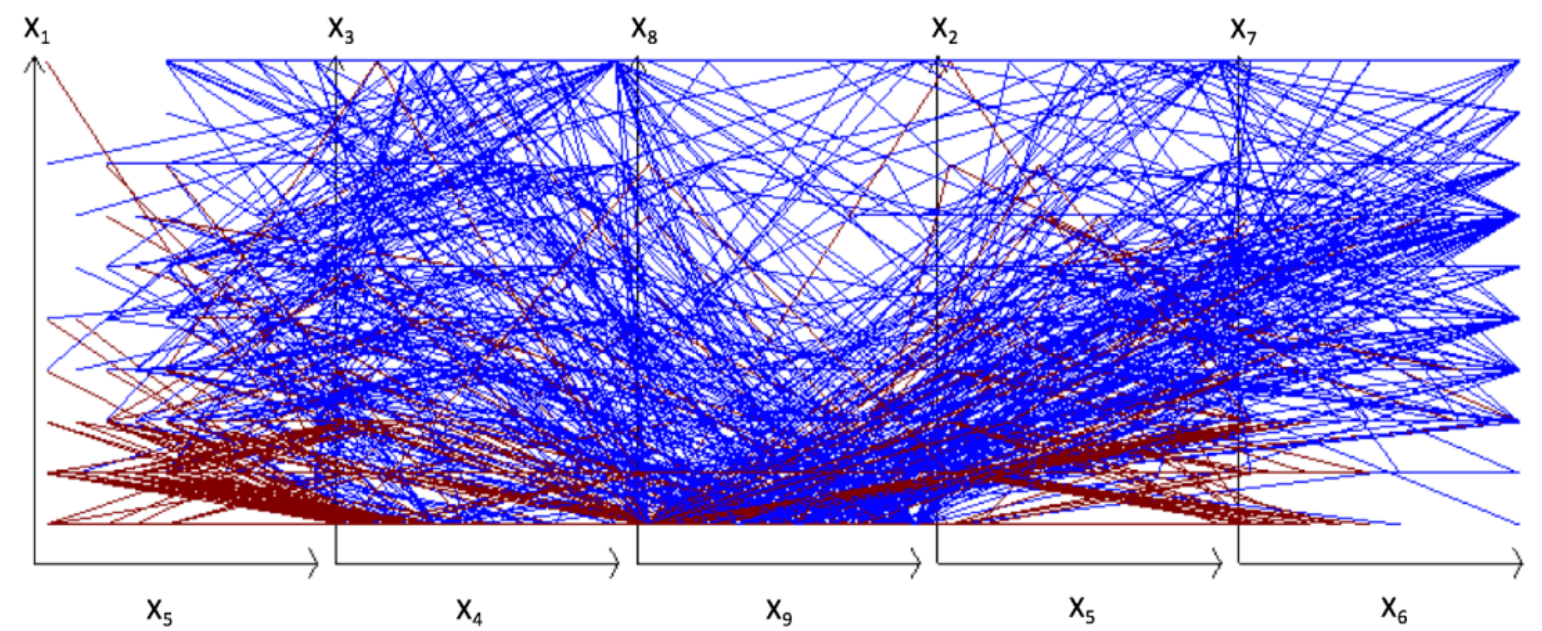

Figure 2: A set of 688 Wisconsin Breast Cancer (WBC) data visualized in SPC as 2-D graphs of 10-D points with benign cases in Red and malignant cases in Blue. 
The challenge is that it is impractical to conduct interactive search of efficient sequences of pairs of coordinates for the large number of sequences. The total number of pairs of $n$ coordinates is the number of combinations $\mathrm{C}(\underline{n}, 2)=n ! /((n-2) ! \cdot 2 !)$, e.g., 45 for $n=10$. Next, there are multiple different sequences of the same set of $\mathrm{n} / 2$ pairs, e.g., $\left(\mathrm{X}_{1}, \mathrm{X}_{2}\right),\left(\mathrm{X}_{3}, \mathrm{X}_{4}\right),\left(\mathrm{X}_{5}, \mathrm{X}_{6}\right), \ldots,\left(\mathrm{X}_{\mathrm{n}}\right.$ $\left.{ }_{1}, X_{n}\right)$ and $\left(X_{5}, X_{6}\right),\left(X_{3}, X_{4}\right),\left(X_{1}, X_{2}\right), \ldots,\left(X_{n-1}, X_{n}\right)$. The number of these sequences (orders) is $(n / 2)$ ! for the same set of $n / 2$ pairs, e.g., (10/2)! $=120$ for $n=10$. Thus, the total number of sequences of all pairs of $n$ coordinates is $(n / 2) ! \cdot \mathrm{C}(n, 2)=(n / 2) ! \cdot n ! /((\mathrm{n}-2) ! \cdot 2 !)$ and for $n=10$ it is $45 \cdot 120=5400$. The analyst cannot observe all of them to find one with the best visual separation of classes. The FSP algorithm resolves this issue by automatic search for the best sequences and presenting only a few best visualizations to the analyst. 


\section{CHAPTER II}

\section{VISUAL KNOWLEDGE DISCOVERY}

\section{FSP Algorithm}

The FSP algorithm:

(a) Filters out less efficient rules and visualizations for supervised classification learning,

(b) Searches for sequences of pairs of coordinates and respective rules that are more efficient for supervised classification learning, and

(c) Presents the SPC visualizations only with better sequences to the analyst.

The main characteristic of FSP is avoiding interactive exploration of the exponential number of alternative sequences with the following major steps:

Step 1: Random generation of sequences of pairs of coordinates $S$;

Step 2: SPC representation of n-D data in sequences $S$ from Step 1;

Step 3: Machine learning process for learning "rectangular" classification rules with high accuracy, precision and recall in sequences $S$ from Step 2;

Step 4: Full automated visualization process: SPC representation of best n-D rules in the best full sequences $S$ of pairs of coordinates $S$ discovered in Step 3;

Step 5: Simplified automated visualization process: SPC representation of best n-D rules in the best subsequences $S$ of pairs of coordinates $S$ discovered in Step 3;

Step 6: Interactive visualization process: an analyst interactively controls and manages produced SPC visualizations.

The approach of this algorithm is in line with [Wilinski, Kovalerchuk, 2017]. It produces efficient classification rules and 3-D visualization of n-D data. In that paper another visualization 
method called Collocated Tripled Coordinates from the class of General Line Coordinates (GLC) is combined with Machine Learning for predicting the investment strategy.

The ideas of steps 1 and 2 already have been explained above. The step 3 uses rules and learning criteria presented below.

Rules and Learning Criteria. The filtering works on a set of rules such as rules (RL1)(RL8) listed below. Each Rule is defined on an n-D point $\mathbf{x}=\left(x_{1}, x_{2}, \ldots, x_{n}\right)$ to be classified to some classes:

$$
\begin{aligned}
& \text { If }\left(x_{\mathrm{i}}, x_{j}\right) \in R_{1} \text { then } \mathbf{x} \in \text { class } 1 \\
& \text { If }\left(x_{\mathrm{i}}, x_{j}\right) \in R_{1} \&\left(x_{k}, x_{m}\right) \in R_{2} \&\left(x_{s}, x_{t}\right) \in R_{3} \text { then } \mathbf{x} \in \text { class } 1 \\
& \text { If }\left(\left(x_{i}, x_{j}\right) \in R_{1} \vee\left(x_{k}, x_{m}\right) \in R_{2}\right) \&\left(x_{s}, x_{t}\right) \in R_{3} \text { then } \mathbf{x} \in \text { class } 1 \\
& \text { If }\left(\left(x_{i}, x_{j}\right) \in R_{1} \vee\left(x_{k}, x_{m}\right) \in R_{2}\right) \&\left(x_{s}, x_{t}\right) \notin R_{3} \text { then } \mathbf{x} \in \text { class } 1 \\
& \text { If }\left(\left(x_{i}, x_{j}\right) \in R_{1} \vee\left(x_{k}, x_{m}\right) \in R_{2}\right) \&\left(x_{s}, x_{t}\right) \notin R_{3} \text { then } \mathbf{x} \in \text { class } 1, \text { else } \mathbf{x} \in \text { class } 2 \\
& \text { If }\left(x_{\mathrm{i}}, x_{j}\right) \in R_{1} \&\left(x_{k}, x_{m}\right) \in R_{2} \&\left(x_{s}, x_{t}\right) \notin R_{3} \text { then } \mathbf{x} \in \text { class } 1 \\
& \text { If }\left(x_{\mathrm{i}}, x_{j}\right) \in R_{1} \&\left(x_{i}, x_{j}\right) \notin R_{2} \&\left(x_{i}, x_{j}\right) \notin R_{3} \text { then } \mathbf{x} \in \text { class } 1 \\
& \text { If } \left.\left(x_{i}, x_{j}\right) \in R_{1} \vee\left(x_{k}, x_{m}\right) \notin R_{2} \vee\left(x_{s}, x_{t}\right) \notin R_{3} \text { then }\right) \mathbf{x} \in \text { class } 1
\end{aligned}
$$

where $R_{1}, R_{2}$ and $R_{3}$ are specific rectangles, in respective pairs of Cartesian coordinates in a given sequence $S$ of pairs of coordinates $\mathrm{S}$, e.g., $R_{1}$ can be in $\left(\mathrm{X}_{1}, \mathrm{X}_{2}\right)$.

The filtering follows common Data Mining/Machine Learning strategy of learning rules on training data and validation on validation data. The quality of learning of classification and expected visualization for rules (RL1)-(RL4), (RL6)-(RL8) is measured by the precision and 
recall of classification of training and validation data, where the precision $\operatorname{Pr}$ is the fraction of the of cases predicted correctly by the Rule to the all predicted cases by the Rule:

$\operatorname{Pr}=\mid\{$ cases predicted correctly by the Rule $\}|/|\{$ all predicted cases by the Rule $\} \mid$.

The precision $\operatorname{Pr}$ for the basic Rule (RL1): If $\left(x_{\mathrm{i}}, x_{j}\right) \in R_{1}$ then $\mathbf{x} \in$ class 1 , is calculated as follows:

$$
\operatorname{Pr}=\frac{n 1\left(R_{1}\right)}{n 1\left(R_{1}\right)+n 2\left(R_{1}\right)}
$$

where $\mathrm{n} 1\left(R_{1}\right)$ is the number of points of class 1 in $R_{1}$ (i.e., the number of correctly classified cases), and $\mathrm{n} 2\left(R_{1}\right)$ is the number of points from class 2 in $R_{1}$ (i.e., the number of misclassified cases). More generally, for any Rule(x) such that

$$
\text { If Rule( }(\mathbf{x}) \text { then } \mathbf{x} \in \text { class } 1
$$

the precision is

$$
\operatorname{Pr}=\frac{n 1(\text { rule })}{n 1(\text { rule })+n 2(\text { rule })}
$$

where $n 1$ (Rule) is the number of points of class 1 that satisfy the if part of the Rule and $n 2$ (Rule) is the number of points of class 2 that satisfy the if the part of the Rule too.

The formula (11) is applicable to all rules (RL1)-(RL8). For example, the precision $\operatorname{Pr}$ for the Rule (RL4) is calculated as follows:

$$
\operatorname{Pr}=\frac{n 1\left(R_{1}\right)+n 1\left(R_{2}\right)-n 1\left(R_{1} \& R_{3}\right)-n 1\left(R_{2} \& R_{3}\right)}{\left.n 1\left(R_{1}\right)+n 1\left(R_{2}\right)-n 1\left(R_{1} \& R_{3}\right)-n 1 R_{2} \& R_{3}\right)+n 2\left(R_{1}\right)+n 2\left(R_{2}\right)-n 2\left(R_{1} \& R_{3}\right)-n 2\left(R_{2} \& R_{3}\right)}
$$

where

$n 1\left(R_{1}\right)$ and $n 1\left(R_{2}\right)$ are the number of points of class 1 in $R_{1}, R_{2}$, respectively, $n 1\left(R_{1} \& R_{3}\right)$ is the number of graphs $\mathbf{x}^{*}$ of the n-D points of class 1 that have 2-D points in both $R_{1}$ and $R_{3}$, $n 1\left(R_{2} \& R_{3}\right)$ is the number of graphs $\mathbf{x}^{*}$ of the n-D points of class 1 that have 2-D points in both $R_{2}$ and $R_{3}$, 
$n 2\left(R_{1} \& R_{3}\right)$ is the number of graphs $\mathbf{x}^{*}$ of the n-D points of class 2 that have 2-D points in both $R_{1}$ and $R_{3}$, $n b\left(R_{2} \& R_{3}\right)$ is the number of graphs $\mathbf{x}^{*}$ of the $\mathrm{n}-\mathrm{D}$ points of class 2 that have 2-D points in both $R_{2}$ and $R_{3}$

Here

$n 1\left(R_{1}\right)+n 1\left(R_{2}\right)-n 1\left(R_{1} \& R_{3}\right)-n 1\left(R_{2} \& R_{3}\right)$ is the number of correctly classified n-D points by Rule (RL4) and $n 2\left(R_{1}\right)+n 2\left(R_{2}\right)-n 2\left(R_{1} \& R_{3}\right)-n 2\left(R_{2} \& R_{3}\right)$ is the number of misclassified $n-\mathrm{D}$ points by this Rule.

We use precision for rules (RL1)-(RL4) and (RL6)-(RL8) instead of accuracy because these rules predict only one class. All cases that do not satisfy the condition of these rules are not classified (refused to be classified). The computing accuracy would require predictions of the class for all cases as it is the case for rules (RL5). Therefore, rules in set of rules (RL5) we use the accuracy for filtering. Note that a high precision Rule from sets of rules (RL1)-(RL4), (RL6)-(RL8) may covers only a few cases, but the precision value does not show it's low coverage. Therefore, we also use the recall

$$
R C=\mid\{\text { cases predicted correctly by the Rule }\}|/|\{\text { all cases }\} \mid
$$

that is a fraction of cases correctly predicted by the Rule to all cases to be predicted.

The Random generation in Step 1 consists of two substeps:

(RS1) Randomly generate a set of pairs of coordinates from coordinates $\mathrm{X}_{1}-\mathrm{X}_{n}$, (RS2) Randomly generate sequence $S_{k}$ for a set of pairs from (RS1).

The Machine Learning process in Step 3 consists of following three substeps:

(ML1) Search for rectangles in each $\left(\mathrm{X}_{i}, \mathrm{X}_{j}\right)$ that maximize precision or accuracy of a Rule from (RL1)-(RL8) on training data for given $S_{k}$,

(ML2) Evaluate this Rule on validation data,

(ML3) Repeat (RS1)-(RS2) and (ML1)-(ML2) in attempt to reach desired precision/accuracy and recall. 
(ML3) Combine promising rules to get a stronger Rule in precision, accuracy and recall.

The Automated Visualization process in Steps 4-5 consists of the following steps:

(AV1) Visualize in SPC most accurate classification results. This includes visualization of only best results.

(AV2) Remove data that are covered by best results in (IV1),

(AV3) Repeat (RS1-RS2) and (ML1-ML3) for remaining data in search for the best classification results.

The Interactive Visualization process in Step 6 is as follows:

(IV1) Substitute automatic search in (ML1) by interactive search where the analysts select rectangles in SPC visualization using GUI.

(IV2) The automatic system supports this interactive process by computing accuracies of rules based on selected rectangles and removing data covered by best results found before the next interactive selection of new rectangles starts.

Fig. 3 shows the overall design of the FSP process.

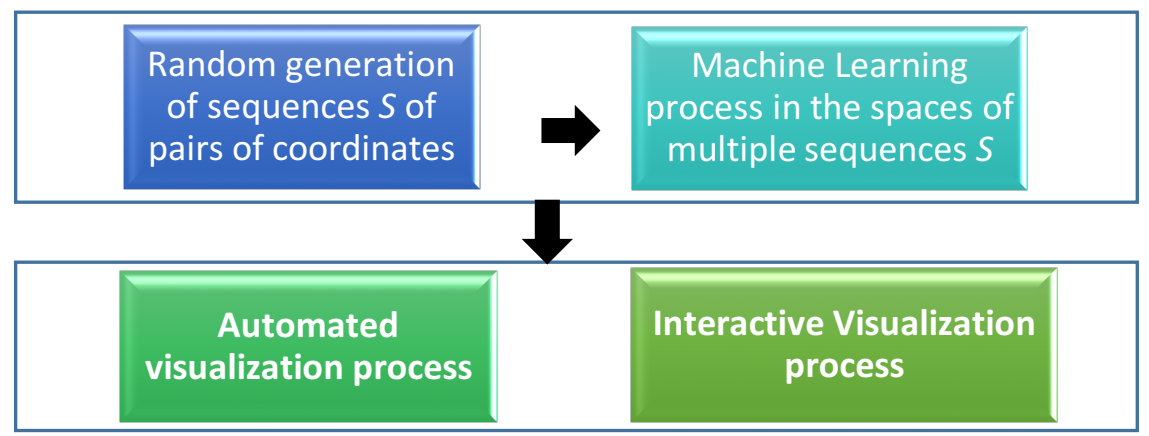

Figure 3: Overall design of the FSP process. 


\section{Frequency Visualization Algorithm}

One of the challenges of SPC visualization for larger data sets is a form of occlusion caused by a limited resolution of data on the screen. It leads to overlap of similar data including complete colocation of some lines. In addition, identical cases will collocate on any visualization at any resolution. Therefore, the task is enhancing the SPC visualization to show frequency of the lines.

The algorithm for this, denoted as FRE algorithm, consists of the following steps:

(F1) For each consecutive pairs of coordinates $\left(\mathrm{X}_{i}, \mathrm{X}_{j}\right),\left(\mathrm{X}_{k}, \mathrm{X}_{m}\right)$ form sets of edges $\left\{E_{q}\right\}$ that are collocated or nearly collocated under some threshold $\mathrm{T}$,

(F2) Count the number of edges $C_{q}(T)$ in each of these sets,

(F3) Draw each such set of edges $E_{q}$ with the adjustable width $w$ :

(a) Equally proportional to the number of edges in $E_{q}$ for each node,

(b) Unequally proportional to the number of edges in $E_{q}$ for each node without adjusting to the data density,

(c) Unequally proportional to the number of edges in $E_{q}$ for each node with adjusting to the data density.

Fig. 4 illustrates the differences between versions (a)-(c) of the algorithm FRE for Red graphs in SPC. The version (a) is "neutral", the analyst can use it when no specific node it set up to explore, Versions (b) and (c) are for exploring specific nodes of interest (e.g. nodes of the discovered classification Rule), because the large width of edge in (a) may occlude that specific node. 


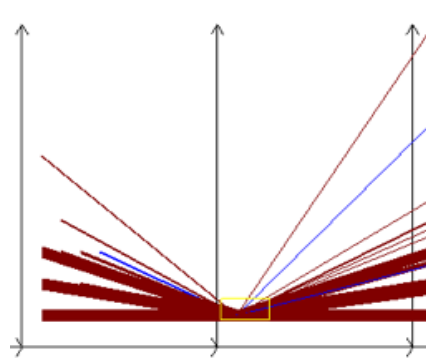

(a) Equal expansion of the width of edges.

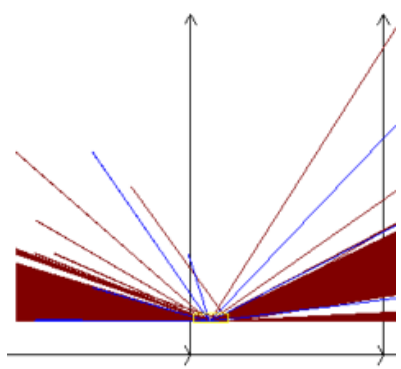

(b) Unequal expansion of the width of edges focused in the given node of graph.

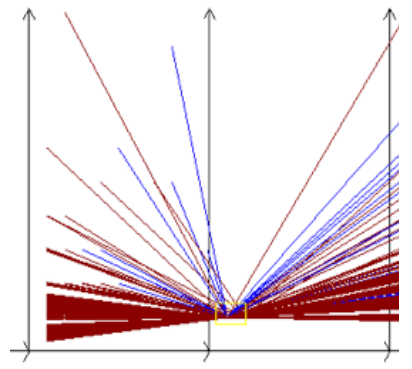

(c) Unequal expansion of the width of edges adjusted to data density focused in the given node of graph.

Figure 4: Alternative frequency visualization. 


\section{CHAPTER III}

\section{EXPERIMENTAL CASE STUDIES}

\section{Experimental Case Study 1}

The computational experiments with the 9-D Wisconsin Breast Cancer (WBC) data, from the UCI Machine Learning repository [3] presented below, show the efficiency of the FSP algorithm. To get the even number of coordinates and 5 pairs of coordinates, the coordinate $\mathrm{X}_{5}$ was duplicated in $\mathrm{X}_{10}$ getting total 10 coordinates.

The discovered patterns were found by the search in the set of rules (RL1)-(RL8). In particular, on WBC data, the FSP algorithm found an efficient sequence of the pairs of the coordinates. This sequence of pairs is $\left(\mathrm{X}_{5}, \mathrm{X}_{1}\right),\left(\mathrm{X}_{4}, \mathrm{X}_{3}\right),\left(\mathrm{X}_{9}, \mathrm{X}_{8}\right),\left(\mathrm{X}_{5}, \mathrm{X}_{2}\right),\left(\mathrm{X}_{6}, \mathrm{X}_{7}\right)$. Here $\mathrm{X}_{5}$ is used in two pairs $\left(\mathrm{X}_{5}, \mathrm{X}_{1}\right)$ and $\left(\mathrm{X}_{5}, \mathrm{X}_{2}\right)$. The SPC visualization with this sequence reveals classification pattern with precision over 90\% in all 11 random 70\%:30\% splits that are presented in Tables 1 and 2. The best precision on the training data is $99.3 \%$, which is accompanied by the high precisions on the validation data $(98.21 \%)$, in one of the $70 \%: 30 \%$ splits of the given data into the training and the validation data.

Table 1 . Number of cases that satisfy the if part of Rule 1 in 11 random 70\%:30\% splits of data.

\begin{tabular}{cccc|ccc}
\hline $\begin{array}{c}70 \%: 30 \% \\
\text { random } \\
\text { data splits }\end{array}$ & \multicolumn{5}{c}{ Number of cases that satisfy if part of the Rule 1 } \\
\cline { 2 - 7 } & training & $\begin{array}{c}\text { Red class } \\
\text { validation }\end{array}$ & total & training & $\begin{array}{c}\text { Blue Class } \\
\text { validation }\end{array}$ & Total \\
\hline 1 & 303 & 122 & 425 & 17 & 4 & 21 \\
2 & 300 & 105 & 405 & 10 & 4 & 14 \\
3 & 290 & 132 & 422 & 20 & 4 & 24 \\
4 & 291 & 110 & 401 & 2 & 2 & 4 \\
5 & 253 & 123 & 376 & 2 & 1 & 3 \\
6 & 297 & 116 & 413 & 13 & 3 & 16 \\
7 & 301 & 121 & 422 & 12 & 2 & 14 \\
8 & 299 & 122 & 421 & 12 & 4 & 16 \\
9 & 282 & 127 & 409 & 10 & 4 & 14 \\
10 & 307 & 116 & 423 & 27 & 7 & 34 \\
11 & 282 & 117 & 399 & 27 & 9 & 36 \\
Average & 291 & 119 & 411 & 14 & 4 & 18 \\
\hline
\end{tabular}


Table 2. Precision, recall and coverage of Rule 1 in 11 random $70 \%: 30 \%$ splits of data.

\begin{tabular}{|c|c|c|c|c|c|c|c|c|}
\hline \multirow{2}{*}{$\begin{array}{c}70 \%: 30 \% \\
\text { random } \\
\text { data splits }\end{array}$} & \multicolumn{2}{|c|}{ Rule precision } & \multicolumn{3}{|c|}{ Rule recall (correct) coverage } & \multirow{2}{*}{$\begin{array}{l}\text { Rule total } \\
\text { coverage of } \\
\text { cases, \% }\end{array}$} & \multicolumn{2}{|c|}{ Rule F measure } \\
\hline & $\begin{array}{c}\text { Training, } \\
\%\end{array}$ & $\begin{array}{c}\text { Validation, } \\
\%\end{array}$ & $\begin{array}{c}\text { Training, } \\
\%\end{array}$ & $\begin{array}{c}\text { Validation, } \\
\%\end{array}$ & $\begin{array}{l}\text { Total, } \\
\%\end{array}$ & & Training & Validation \\
\hline 1 & 94.06 & 96.82 & 44.04 & 17.73 & 61.77 & 64.83 & 0.5999 & 0.2997 \\
\hline 2 & 96.77 & 96.33 & 43.6 & 15.26 & 58.86 & 60.9 & 0.6012 & 0.2635 \\
\hline 3 & 93.5 & 97.05 & 42.15 & 19.19 & 61.34 & 64.83 & 0.5811 & 0.3204 \\
\hline 4 & 99.3 & 98.21 & 42.3 & 15.99 & 58.29 & 58.87 & 0.5933 & 0.2750 \\
\hline 5 & 98.41 & 99.19 & 36.77 & 17.88 & 54.65 & 55.09 & 0.5354 & 0.3030 \\
\hline 6 & 95.8 & 97.47 & 43.17 & 16.86 & 60.03 & 62.35 & 0.5952 & 0.2875 \\
\hline 7 & 96.16 & 98.37 & 43.75 & 17.59 & 61.34 & 63.37 & 0.6014 & 0.2984 \\
\hline 8 & 96.14 & 96.82 & 43.46 & 17.73 & 61.19 & 63.52 & 0.5986 & 0.2997 \\
\hline 9 & 96.57 & 96.94 & 40.99 & 18.46 & 59.45 & 61.48 & 0.5755 & 0.3101 \\
\hline 10 & 91.91 & 94.3 & 44.62 & 16.86 & 61.48 & 66.42 & 0.6008 & 0.2861 \\
\hline 11 & 91.26 & 92.85 & 40.99 & 17.01 & 58 & 63.23 & 0.5657 & 0.2875 \\
\hline Average & 95.44 & 96.76 & 42.3 & 17.3 & 59.6 & 62.35 & 0.5862 & 0.2935 \\
\hline
\end{tabular}

The discovered rules in Tables 1 and 2 belong to the set of rules (RL7). The first Rule in Table 1 that we denote as WBC Rule 1 is:

$$
\text { If }\left(x_{9}, x_{8}\right) \in R_{1} \&\left(x_{6}, x_{7}\right) \notin R_{2} \&\left(x_{6}, x_{7}\right) \notin R_{3} \text { then } \mathbf{x} \in \text { class } 1 \text { (Red, Benign) }
$$

where $R_{1}, R_{2}$, and $R_{3}$ are specific rectangles, in respective pairs of Cartesian coordinates $\left(\mathrm{X}_{8}, \mathrm{X}_{9}\right)$ and $\left(\mathrm{X}_{6}, \mathrm{X}_{7}\right)$. Table 3 shows the parameters of $R_{1}, R_{2}$ and $R_{3}$ in the normalized coordinates.

Table 3. Parameters of rectangles $\mathrm{R}_{1}-\mathrm{R}_{3}$.

\begin{tabular}{ccccc}
\hline Rectangle & Left & Right & Parameters & Bottom \\
\hline$R_{1}$ in $\left(\mathrm{X}_{9}, \mathrm{X}_{8}\right)$ & 0.0020 & 0.1402 & 0.0734 & 0.1028 \\
$R_{2}$ in $\left(\mathrm{X}_{6}, \mathrm{X}_{7}\right)$ & 0.7214 & 1.001 & 0.3484 & 1.001 \\
$R_{3}$ in $\left(\mathrm{X}_{6}, \mathrm{X}_{7}\right)$ & 0.0081 & 0.6325 & 0.6014 & 1.001 \\
\hline
\end{tabular}

This Rule, with a random 70\%:30\% data split into the training and the validation data, has the precision of $94.6 \%$ on the training data, and $96.82 \%$ on the validation data. Figs. 5 and 6 show its rectangles $R_{1}, R_{2}$ and $R_{3}$ drawn in the SPC as magenta boxes. The difference between these figures is that Fig. 5 shows all graphs that go through rectangle $R_{1}$ (have node $\left(x_{9}, x_{8}\right)$ in $R_{1}$ ), but Fig. 6 shows only graphs that in addition do not go rectangles $R_{2}$ and $R_{3}$. (do not have node $\left(x_{6}, x_{7}\right)$ in $R_{2}$ and $\left.R_{3}\right)$. Thus, Fig. 6 shows only graphs that satisfy Rule 1. 


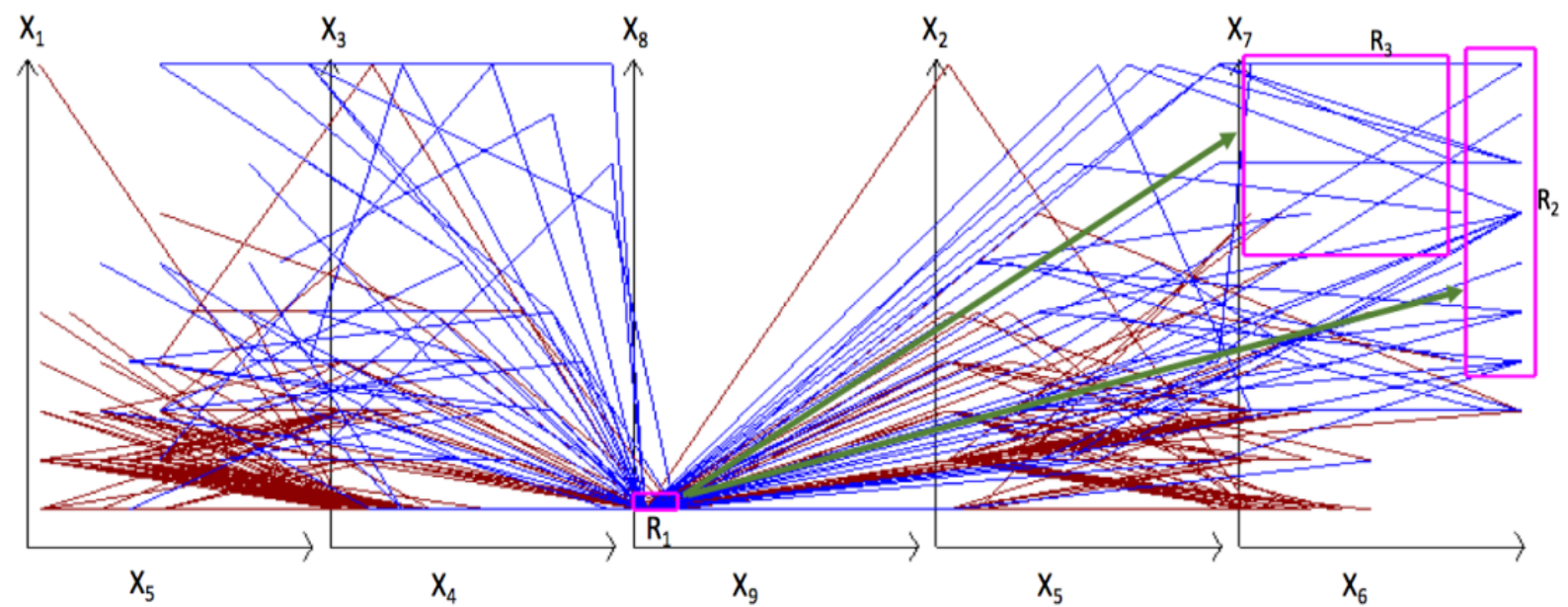

Figure 5: WBC data in SPC as graphs representing 10-D points that go through R1 without showing frequency of cases. Magenta boxes show rectangles R1-R3.

In Fig. 5, the width of the lines are not adjusted their frequency, but in Fig. 6 the width of the lines is adjusted to their frequencies. The Rule 1 covers $64.8 \%$ of all given 9-D points: 446 cases out of 688 cases (425 Red cases and 21 Blue cases) with the recall value $61.77 \%(425 / 688)$ as shown in Table 1. Among these 446 cases 303 Red and 17 Blue cases belong to training data and 122 Red and 4 Blue cases belong to validation data.

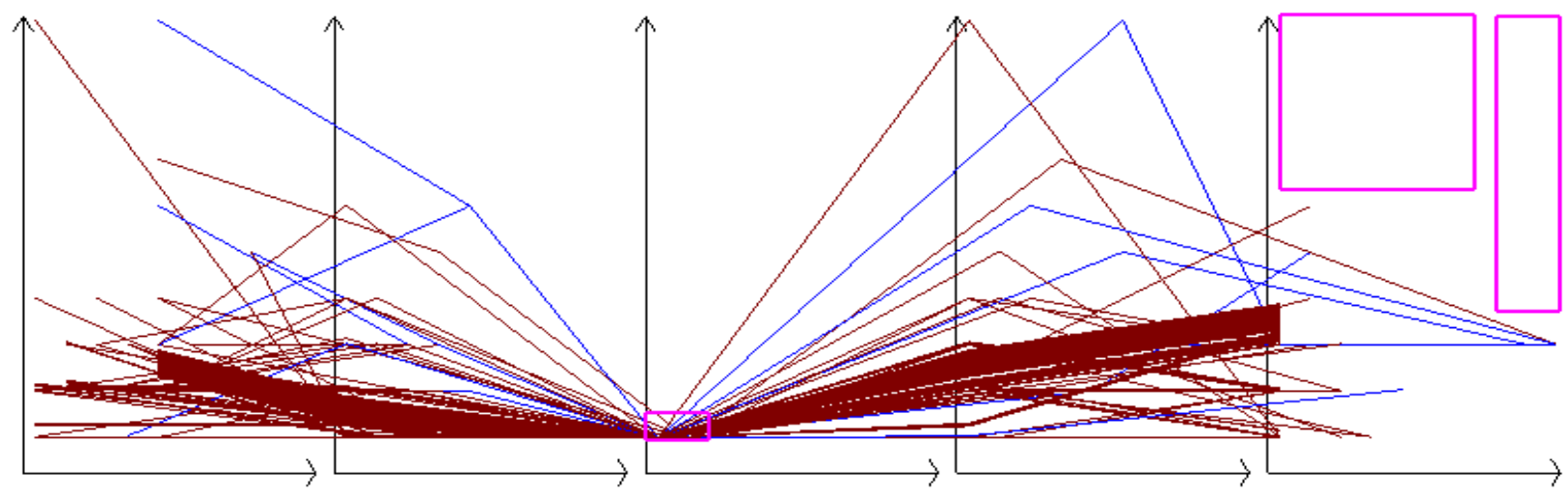

Figure 6: WBC data in SPC as graphs representing 10-D points that satisfy Rule 1 (go through R1 and not coming to R2 and R3 shown in magenta. Wide Red lines show the frequency of Red cases.

The cases covered by Rule 1 do not include only the 5.13\% (23 cases) of the Red data, but include the $91.25 \%$ (219 cases) of the Blue data. This Rule covers only cases found in $R_{1}$ 
that do not come to $R_{2}$ or $R_{3}$. Rule 1 refused to predict other cases. Those other cases are dominantly Blues (see Fig. 7) and must be covered by other rules. The WBC Rule 1 uses only 4 coordinates that form two pairs $\left(\mathrm{X}_{9}, \mathrm{X}_{8}\right)$ and $\left(\mathrm{X}_{6}, \mathrm{X}_{7}\right)$ therefore we can simplify the visualization of this Rule by showing only them in SPC. It is done in Fig. 8 where each 4-D points is visualized losslessly as a single line. The advantage of this visualization is that it is easy to see and communicate to the medical experts. The medical expert can easily understand this Rule because it simply says that two attributes $x_{8}$ and $x_{9}$ must be in some limits identified in Table 3 and two other attributes $x_{6}$ and $x_{7}$ must not have values in some intervals that are shown visually in Fig. 8.

This allows the medical experts to analyze the consistency of this Rule with the other medical domain knowledge, which is extremely difficult for the ML discrimination functions, which are "black" boxes or complex mathematical formulas.

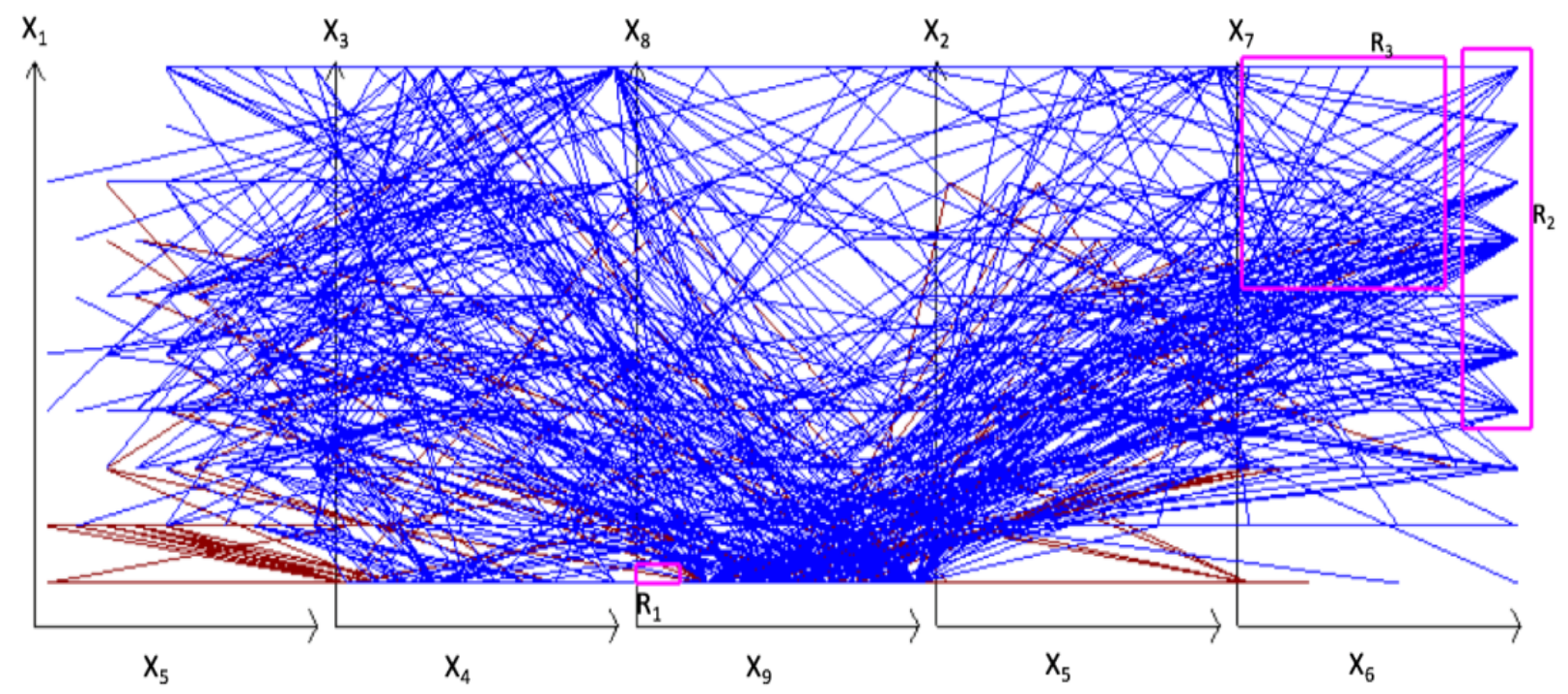

Figure 7: Remaining WBC cases not covered by Rule 1 (dominated by Blue class). 


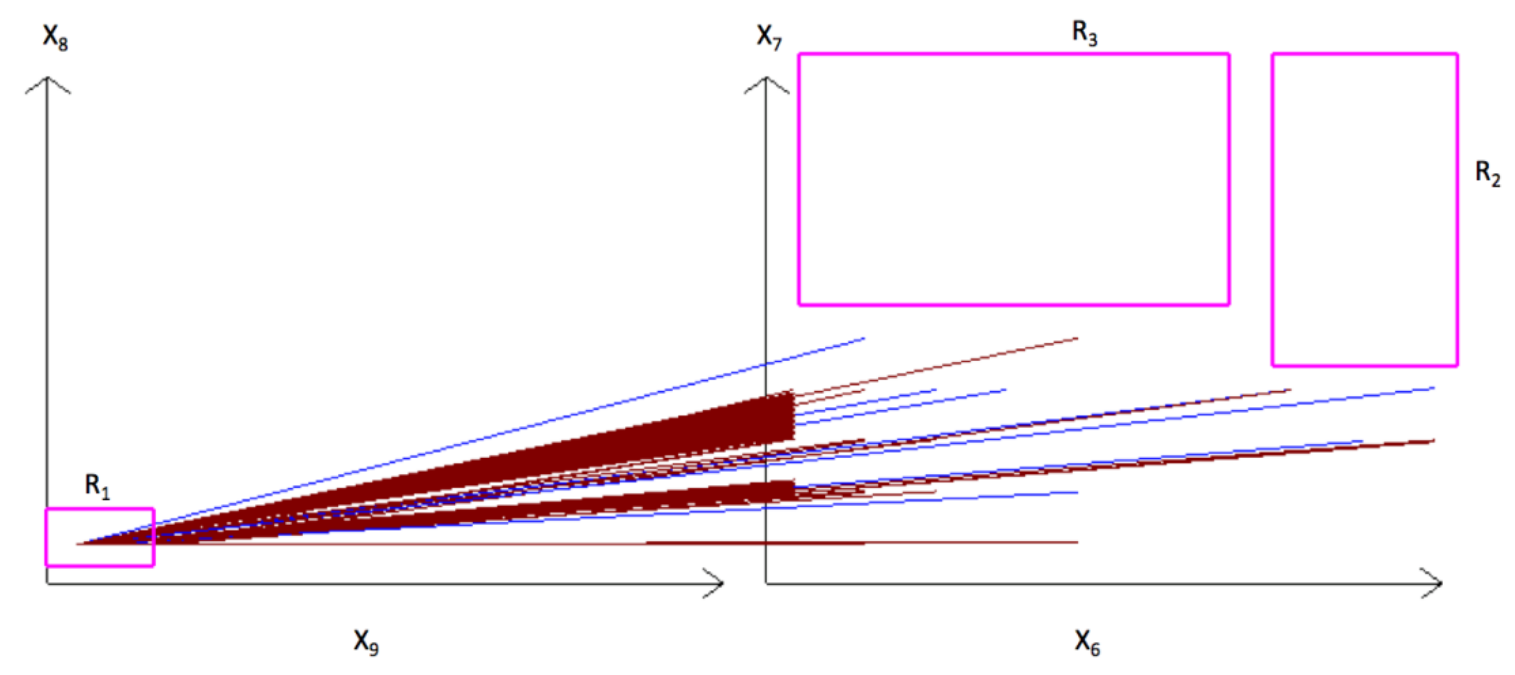

Figure 8: WBC data in 4-D SPC as graphs in coordinates $\left(\mathrm{X}_{9}, \mathrm{X}_{8}\right)$ and $\left(\mathrm{X}_{6}, \mathrm{X}_{7}\right)$ that are used by Rule 1, i.e., WBC cases that go through $\mathrm{R}_{1}$ and not go to $\mathrm{R}_{2}$ and $\mathrm{R}_{3}$ in these coordinates.

The simple WBC Rule 2 classifies all remaining cases (not covered by Rule 1) to class 2 is

$$
\begin{aligned}
& \text { If }\left(x_{8}, x_{9}\right) \in R_{1} \&\left(x_{6}, x_{7}\right) \notin R_{2} \&\left(x_{6}, x_{7}\right) \notin R_{3} \\
& \text { then } \mathbf{x} \in \text { class } 1 \text { (Red, Benign) else } \mathbf{x} \in \text { class } 2 \text { (Blue, Malignant) }
\end{aligned}
$$

This Rule classifies all cases that are either in $R_{2}$ or in $R_{3}$ or not in $R_{1}$ as Blue. This Rule has accuracy $93.60 \%(425+219) / 688)$ on all 688 cases as the confusion matrix shows in Table 4 . Its accuracy on training data is $92.53 \%$ and on validation data it is $96.11 \%$ computed from respective confusion matrixes shown in Table 4.

Table 4. Confusion matrixes of WBC Rule 2.

\begin{tabular}{l|lll}
\hline $\begin{array}{l}\text { Actual } \\
\text { class }\end{array}$ & \multicolumn{3}{|l}{$\begin{array}{l}\text { Confusion matrix on } \\
\text { all } 688 \text { cases }\end{array}$} \\
& \multicolumn{3}{|c}{ Predicted class } \\
& Red & Blue & Total \\
Red & 425 & 23 & 448 \\
Blue & 21 & 219 & 240 \\
Total & 446 & 242 & 688 \\
\hline
\end{tabular}

\begin{tabular}{lll}
\hline \multicolumn{2}{l}{ Confusion matrix on } \\
$70 \%$ of training cases \\
$(482)$ & & \\
Predicted class & \\
Red & Blue & Total \\
303 & 19 & 322 \\
17 & 143 & 160 \\
320 & 162 & 482 \\
\hline
\end{tabular}

Confusion matrix on $30 \%$ on validation cases (206)

Predicted class

Red Blue Total

$\begin{array}{lll}122 & 4 & 126\end{array}$

$\begin{array}{lll}4 & 76 & 80\end{array}$

\begin{tabular}{l}
$126 \quad 89 \quad 206$ \\
\hline
\end{tabular}


We found a better WBC Rule 3 by searching the rectangles with highest density of Blue cases:

$$
\text { If }\left(\left(x_{1}, x_{6}\right) \in R_{4} \vee\left(x_{3}, x_{5}\right) \in R_{5}\right) \&\left(x_{2}, x_{5}\right) \notin R_{6} \text { then } \mathbf{x} \in \text { class } 2 \text { ( Blue ) }
$$

This Rule is of type of rules (RL4), but with the conclusion that $\mathbf{x}$ belongs to class 2, not class 1 . Table 5 identifies the rectangles $\mathrm{R}_{4}-\mathrm{R}_{6}$ involved in this Rule. WBC Rule 3 covers the 226 cases from class 2 (Blue), and the 12 cases from class 1 (Red) with the total precision of $94.95 \%$. The combined Rule1 and Rule 3 is as follows:

If Rule1(x) then $\mathbf{x} \in$ Red, else if Rule3(x) then $\mathbf{x} \in$ Blue, else refuse to classify $\mathbf{x}$

The precision, recall and coverage of this Rule relative to all cases are $95.18 \%, 94.62 \%$, and 99.42\% (Fig. 9). It is the performance details are in the confusion matrix in Table 6.

Table 5. Parameters of rectangles $\mathrm{R}_{4}-\mathrm{R}_{6}$ in normalized coordinates.

\begin{tabular}{ccccc}
\hline Rectangle & & \multicolumn{2}{c}{ Parameters } & Top \\
\cline { 2 - 5 }$R_{4}$ in $\left(X_{1}, X_{6}\right)$ & Left & Right & Bottom & 1.001 \\
$R_{5}$ in $\left(X_{3}, X_{5}\right)$ & 0.0010 & 0.9712 & 0.4180 & 0.7261 \\
$R_{6}$ in $\left(X_{2}, X_{5}\right)$ & 0.0000 & 0.9881 & 0.3154 & 0.3153 \\
\hline
\end{tabular}

Table 6. Confusion matrix of combined Rules 1 and 3 on all 688 cases.

\begin{tabular}{ccccc}
\hline & Predicted Red class & Predicted Blue class & Refusal & Total \\
\cline { 2 - 5 } Actual Red class & 425 & 12 & 2 & 437 \\
Actual Blue class & 21 & 226 & 2 & 247 \\
Total & 446 & 238 & 4 & 688 \\
\hline
\end{tabular}

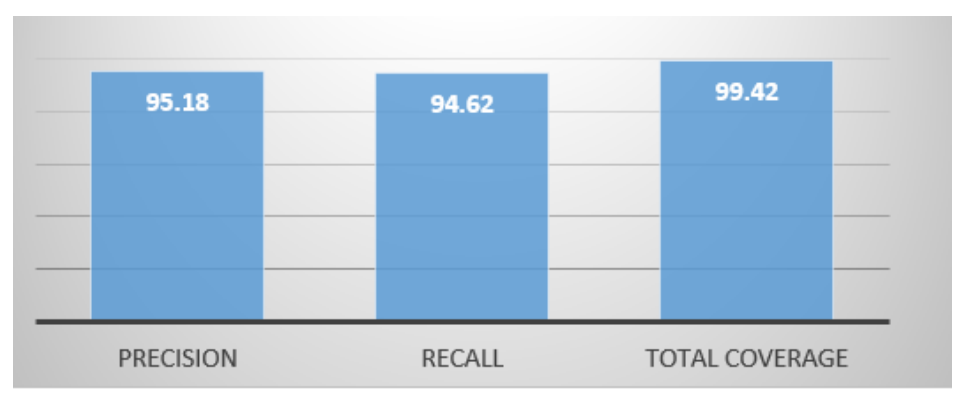

Figure 9: The precision, recall and total coverage of combined Rule 1 and Rule 3 


\section{Experimental Case Study 2}

The computational experiments with the 33-D Ionosphere data from the UCI Machine Learning repository [3] also show the efficiency of the FSP algorithm. To get the even number of coordinates and 17 pairs of coordinates, the algorithm in each epoch will select randomly the coordinate that will serve as coordinate $X_{34}$. In the following results $X_{34}$ is a copy of $X_{10}$. The discovered patterns also were found by the search in the set of "rectangular" rules (RL1)-(RL8). In particular, on Ionosphere data, the FSP algorithm found an efficient sequence of pairs of coordinates: $\left(\mathrm{X}_{5}, \mathrm{X}_{26}\right),\left(\mathrm{X}_{27}, \mathrm{X}_{16}\right),\left(\mathrm{X}_{4}, \mathrm{X}_{11}\right),\left(\mathrm{X}_{18}, \mathrm{X}_{24}\right),\left(\mathrm{X}_{28}, \mathrm{X}_{31}\right),\left(\mathrm{X}_{10}, \mathrm{X}_{3}\right),\left(\mathrm{X}_{23}, \mathrm{X}_{8}\right),\left(\mathrm{X}_{22}, \mathrm{X}_{30}\right)$, $\left(X_{21}, X_{10}\right),\left(X_{17},\right),\left(X_{15}, X_{33}\right),\left(X_{29}, X_{20}\right),\left(X_{9}, X_{6}\right),\left(X_{32}, X_{16}\right),\left(X_{1}, X_{25}\right),\left(X_{12}, X_{14}\right),\left(X_{19}, X_{7}\right)$. Here $\mathrm{X}_{10}$ is repeated in pairs $\left(\mathrm{X}_{10}, \mathrm{X}_{3}\right)$ and $\left(\mathrm{X}_{21}, \mathrm{X}_{10}\right)$. Similarly to the Case Study 1 , the SPC visualization, with this sequence, reveals the visual classification pattern of precision of over 90\% in all the 11 random 70\%:30\% splits of data into the training and the validation data (see Tables 7 and 8). The best precision on the training data is $98.36 \%$ with $100 \%$ precision on the validation data in one of these 70\%:30\% splits of the given data. The discovered rules in Tables 7 and 8 belong to the set of rules (RL4). The first Rule in Table 7 that we denote as Ionosphere

Rule 1 is:

$$
\text { If }\left[\left(x_{4}, x_{11}\right) \in R_{1} \vee\left(x_{5}, x_{26}\right) \in R_{8} \vee\left(x_{21}, x_{10}\right) \in R_{9} \vee\left(x_{19}, x_{7}\right) \in R_{10}\right] \&
$$

$\left[\left(x_{27}, x_{16}\right) \notin R_{3} \&\left(x_{28}, x_{31}\right) \notin R_{6} \&\left(x_{23}, x_{8}\right) \notin R_{4} \&\left(x_{17}, x_{2}\right) \notin R_{5} \&\left(x_{15}, x_{33}\right) \notin R_{7} \&\left(x_{9}, x_{6}\right) \notin R_{2}\right]$ then $\mathrm{x} \in$ class 1 (Red,Good)

where $R_{1}, R_{2}, R_{3}, R_{4}, R_{5}, R_{6}, R_{7}, R_{8}, R_{9}$, and $R_{10}$ are specific rectangles, in respective pairs of Cartesian coordinates $\left(\mathrm{X}_{4}, \mathrm{X}_{11}\right),\left(\mathrm{X}_{9}, \mathrm{X}_{6}\right),\left(\mathrm{X}_{27}, \mathrm{X}_{16}\right),\left(\mathrm{X}_{23}, \mathrm{X}_{8}\right),\left(\mathrm{X}_{17}, \mathrm{X}_{2}\right),\left(\mathrm{X}_{28}, \mathrm{X}_{31}\right),\left(\mathrm{X}_{15}, \mathrm{X}_{33}\right)$, $\left(\mathrm{X}_{5}, \mathrm{X}_{26}\right),\left(\mathrm{X}_{21}, \mathrm{X}_{10}\right)$, and $\left(\mathrm{X}_{19}, \mathrm{X}_{7}\right)$. Table 9 shows the parameters of $R_{1}-R_{10}$ in the normalized

coordinates. This Rule, with a random 70\%:30\% data split into the training and validation data, 
has the precision $98.36 \%$ on the training data and $100 \%$ on validation data. Figs. 10 and 11 show its rectangles $R_{1}-R_{10}$ in the SPCs as magenta boxes and cases that satisfy Rule 1 in Fig 11 and all cases in Fig. 10. Fig. 12 shows the remaining cases.

Table 7. Number of cases that satisfy the Ionosphere Rule 1 in 11 random 70\%:30\% splits of data

\begin{tabular}{ccccccc}
\hline $\begin{array}{c}70 \%: 30 \% \\
\text { random } \\
\text { data splits }\end{array}$ & training & $\begin{array}{c}\text { Number of cases that satisfy if part of the Rule 1 } \\
\text { Red class } \\
\text { validation }\end{array}$ & total & training & $\begin{array}{c}\text { Blue Class } \\
\text { validation }\end{array}$ & Total \\
\hline 1 & 180 & 45 & 225 & 3 & 0 & 3 \\
2 & 172 & 52 & 224 & 8 & 2 & 10 \\
3 & 133 & 92 & 225 & 7 & 3 & 10 \\
4 & 129 & 95 & 224 & 6 & 2 & 8 \\
5 & 200 & 24 & 224 & 9 & 0 & 3 \\
6 & 160 & 65 & 225 & 10 & 2 & 12 \\
7 & 183 & 42 & 225 & 11 & 1 & 13 \\
8 & 158 & 67 & 225 & 11 & 2 & 13 \\
9 & 191 & 34 & 225 & 13 & 1 & 14 \\
10 & 184 & 37 & 221 & 7 & 0 & 7 \\
11 & 157 & 66 & 223 & 12 & 2 & 14 \\
Average & 170 & 54 & 224 & 9 & 1 & 9 \\
\hline
\end{tabular}

Table 8. Precision, recall and coverage of Ionosphere Rule 1 in 11 random 70\%:30\% splits of data.

\begin{tabular}{|c|c|c|c|c|c|c|c|c|}
\hline \multirow{2}{*}{$\begin{array}{c}70 \%: 30 \% \\
\text { random } \\
\text { data splits }\end{array}$} & \multicolumn{2}{|c|}{ Rule precision } & \multicolumn{3}{|c|}{ Rule recall (correct) coverage } & \multirow{2}{*}{$\begin{array}{l}\text { Rule total } \\
\text { coverage of } \\
\text { cases, } \%\end{array}$} & \multicolumn{2}{|c|}{ Rule F measure } \\
\hline & $\begin{array}{c}\text { Training, } \\
\% \\
\end{array}$ & $\begin{array}{c}\text { Validation, } \\
\%\end{array}$ & $\begin{array}{c}\text { Training, } \\
\%\end{array}$ & $\begin{array}{c}\text { Validation, } \\
\% \\
\end{array}$ & $\begin{array}{c}\text { Total, } \\
\%\end{array}$ & & $\begin{array}{c}\text { Validation, } \\
\% \\
\end{array}$ & $\begin{array}{c}\text { Total, } \\
\%\end{array}$ \\
\hline 1 & 98.36 & 100 & 51.28 & 12.82 & 64.1 & 64.95 & 0.6741 & 0.2273 \\
\hline 2 & 95.55 & 96.29 & 49 & 14.81 & 63.81 & 66.6 & 0.6478 & 0.2567 \\
\hline 3 & 95 & 96.84 & 37.89 & 26.21 & 64 & 66.95 & 0.5417 & 0.4125 \\
\hline 4 & 95.5 & 97.93 & 36.75 & 27.06 & 63.81 & 66.09 & 0.5308 & 0.4240 \\
\hline 5 & 95.69 & 100 & 56.98 & 6.83 & 63.81 & 64.67 & 0.7143 & 0.1279 \\
\hline 6 & 94.1 & 97.01 & 45.58 & 18.51 & 64.1 & 67.52 & 0.6141 & 0.3109 \\
\hline 7 & 94.32 & 97.67 & 52.13 & 11.96 & 64.1 & 67.80 & 0.6715 & 0.2131 \\
\hline 8 & 93.491 & 97.1 & 45.01 & 19.08 & 64.09 & 67.80 & 0.6077 & 0.3189 \\
\hline 9 & 93.62 & 97.14 & 54.41 & 9.68 & 64.1 & 68.09 & 0.6882 & 0.1761 \\
\hline 10 & 96.33 & 100 & 52.421 & 10.54 & 62.96 & 64.95 & 0.6789 & 0.1907 \\
\hline 11 & 92.89 & 97.05 & 44.72 & 18.8 & 63.53 & 67.52 & 0.6037 & 0.3150 \\
\hline Average & 94.99 & 97.93 & 48.43 & 15.38 & 63.81 & 66.38 & 0.6339 & 0.2703 \\
\hline
\end{tabular}

Table 9. Parameters of rectangles R4-R6 in normalized coordinates.

\begin{tabular}{ccccc}
\hline Rectangle & Left & Right & Parameters & Top \\
\cline { 2 - 5 } $\mathrm{R}_{1}$ in $\left(X_{4}, X_{11}\right)$ & 0.23912 & 1.001 & 0.663667 & 0.999667 \\
$\mathrm{R}_{2}$ in $\left(\mathrm{X}_{9}, \mathrm{X}_{6}\right)$ & 0.1979 & 0.99869 & 0 & 0.0403333 \\
$R_{3}$ in $\left(\mathrm{X}_{27}, \mathrm{X}_{16}\right)$ & 0.130225 & 1.001 & 0 & 0.0256667 \\
$R_{4}$ in $\left(\mathrm{X}_{23}, \mathrm{X}_{8}\right)$ & 0.29087 & 0.58943 & 1.001 & 0.946 \\
$R_{5}$ in $\left(\mathrm{X}_{17}, \mathrm{X}_{2}\right)$ & 0.40433 & 0.9998 & 0 & 0.022 \\
$R_{6}$ in $\left(\mathrm{X}_{28}, \mathrm{X}_{31}\right)$ & 0.30765 & 1.001 & 0 & 0.0366667 \\
$R_{7}$ in $\left(\mathrm{X}_{15}, \mathrm{X}_{33}\right)$ & 0.25733 & 1.001 & 0.960667 & 0.998667 \\
$R_{8}$ in $\left(\mathrm{X}_{5}, \mathrm{X}_{26}\right)$ & 0.125675 & 0.575667 & 0.575667 & 0.645333 \\
$R_{9}$ in $\left(\mathrm{X}_{21}, \mathrm{X}_{10}\right)$ & 0.25278 & 0.50868 & 0.0476667 & 0.0843333 \\
$R_{10}$ in $\left(\mathrm{X}_{19}, \mathrm{X}_{7}\right)$ & 0.4203 & 0.8254 & 0.194333 & 0.432667 \\
\hline
\end{tabular}


The Rule 1 uses only 20 coordinates that form 10 pairs $\left(X_{5}, X_{26}\right),\left(X_{27}, X_{16}\right),\left(X_{4}, X_{11}\right)$, $\left(\mathrm{X}_{28}, \mathrm{X}_{31}\right),\left(\mathrm{X}_{23}, \mathrm{X}_{8}\right),\left(\mathrm{X}_{21}, \mathrm{X}_{10}\right),\left(\mathrm{X}_{17}, \mathrm{X}_{2}\right),\left(\mathrm{X}_{15}, \mathrm{X}_{33}\right),\left(\mathrm{X}_{9}, \mathrm{X}_{6}\right)$ and $\left(\mathrm{X}_{19}, \mathrm{X}_{7}\right)$. Therefore, we simplify its visualization by showing only them in the SPCs (see Figs. 13-15) with the lossless visualization of each of the 20-D points, as a single polyline. The simple Ionosphere Rule 2 classifies all remaining cases (not covered by Rule 1) to class 2 is

$$
\text { If }\left[\left(x_{4}, x_{11}\right) \in R_{1} \vee\left(x_{5}, x_{26}\right) \in R_{8} \vee\left(x_{21}, x_{10}\right) \in R_{9} \vee\left(x_{19}, x_{7}\right) \in R_{10}\right] \&
$$

$\left[\left(x_{27}, x_{16}\right) \notin R_{3} \&\left(x_{28}, x_{31}\right) \notin R_{6} \&\left(x_{23}, x_{8}\right) \notin R_{4} \&\left(x_{17}, x_{2}\right) \notin R_{5} \&\left(x_{15}, x_{33}\right) \notin R_{7} \&\left(x_{9}, x_{6}\right) \notin R_{2}\right]$ then $\mathrm{x} \in$ class 1 (Red, Good) else $\mathrm{x} \in$ class 2 (Blue, Bad)

This Rule classifies all the cases rejected by Rule 1 as Blue with $99.14 \%$ accuracy on all the 351 cases $(225+123) / 351)$, see the confusion matrix in Table 10. Its accuracy on training data is $98.78 \%$ and $100 \%$ on the validation data based on the confusion matrixes in Table 10 . 


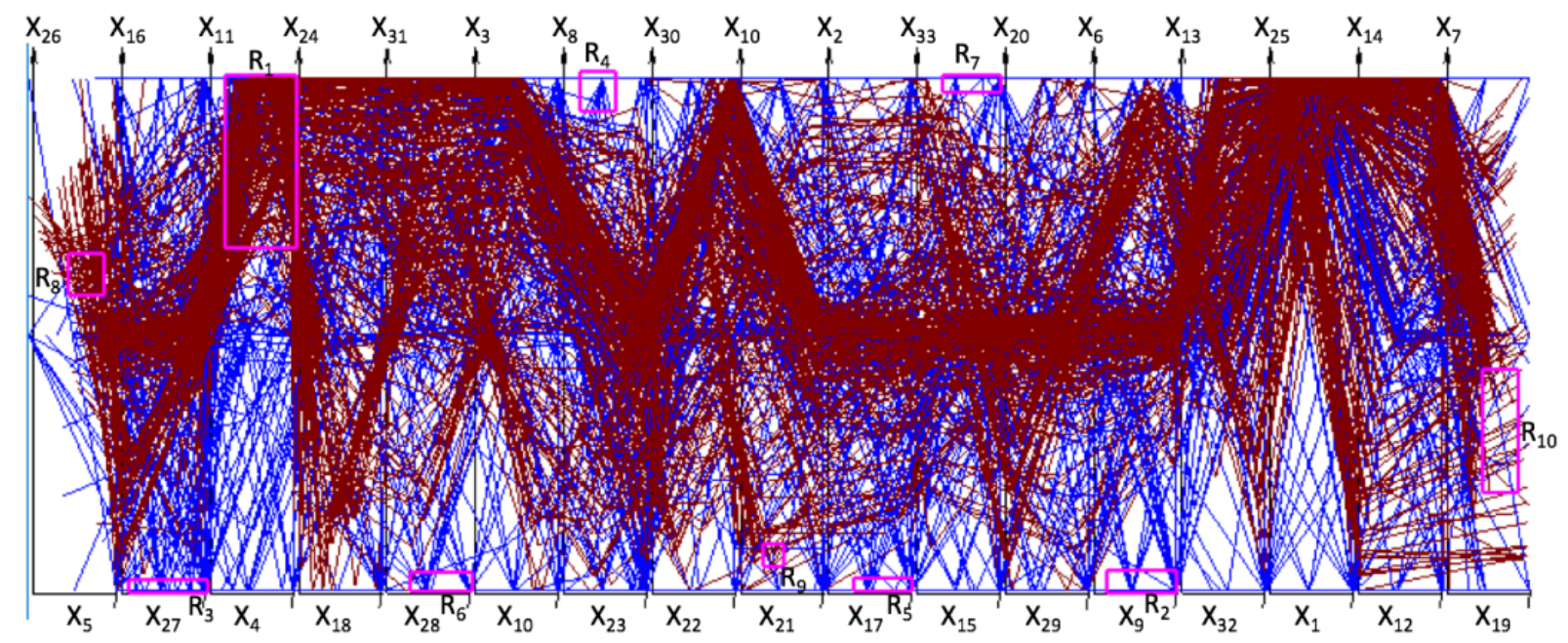

Figure 10: 351 Ionosphere cases in the SPCs as graphs of 34-D points (good cases in Red and bad cases in Blue). Rectangles that are used in Ionosphere Rule 1 are in magenta.

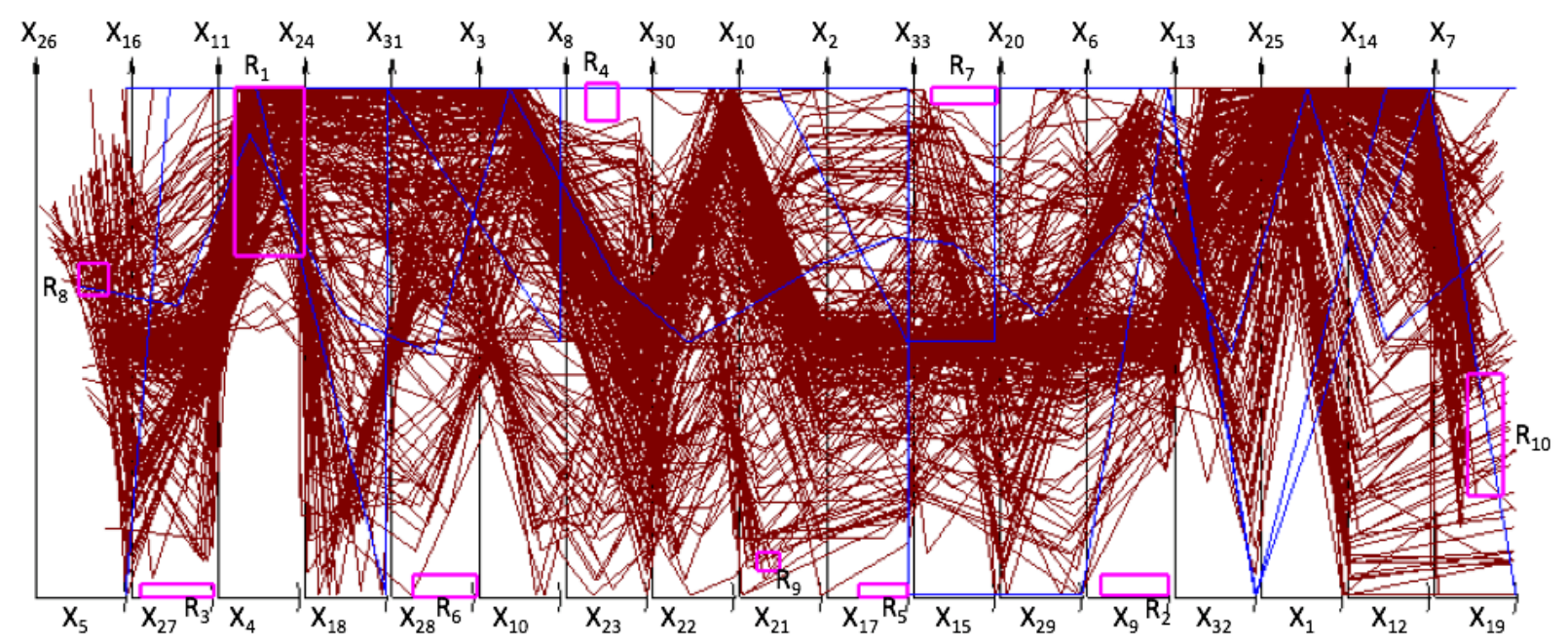

Figure 11: 34-D Ionosphere cases covered by Ionosphere Rule 1. Rectangles from Rule 1 are in magenta.

Table 10. Confusion matrixes of the Ionosphere Rule 2.

\begin{tabular}{l|lll}
\hline $\begin{array}{l}\text { Actual } \\
\text { class }\end{array}$ & \multicolumn{3}{|l}{ Confusion matrix on } \\
& \multicolumn{3}{|l}{ Predicted class } \\
& Red & Blue & Total \\
Red & 225 & 3 & 228 \\
Blue & 0 & 123 & 123 \\
Total & 225 & 126 & 351 \\
\hline
\end{tabular}

\begin{tabular}{lll}
\hline \multicolumn{3}{l}{ Confusion matrix on } \\
training data & $(246,70 \%)$ \\
\multicolumn{3}{l}{ Predicted class } \\
Red & Blue & Total \\
180 & 3 & 183 \\
0 & 63 & 63 \\
180 & 66 & 246 \\
\hline
\end{tabular}

\begin{tabular}{lll}
\hline \multicolumn{3}{l}{ Confusion matrix on } \\
\multicolumn{3}{l}{ validation data } \\
\multicolumn{2}{l}{ Predicted class } \\
Red & Blue & Total \\
45 & 0 & 45 \\
0 & 60 & 60 \\
45 & 60 & 105 \\
\hline
\end{tabular}




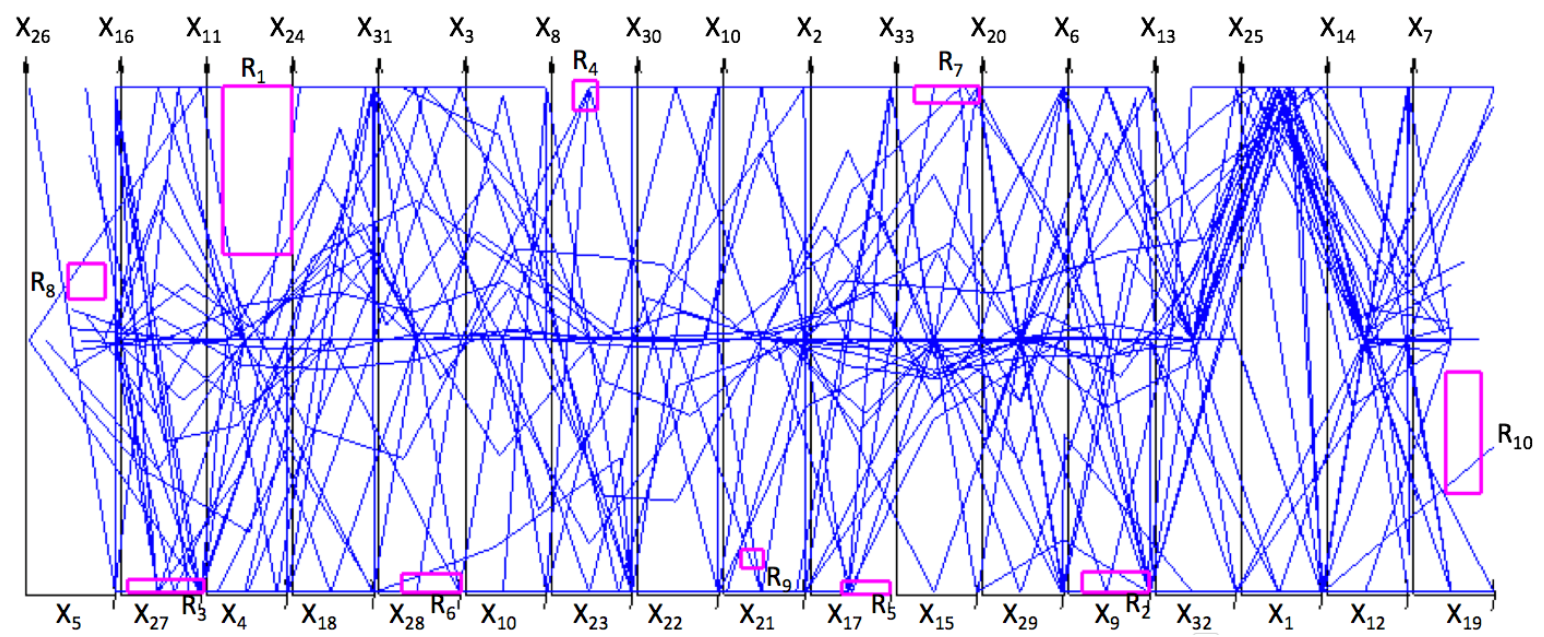

Figure 12: Remaining Ionosphere cases (cases not covered by Ionosphere Rule 1).

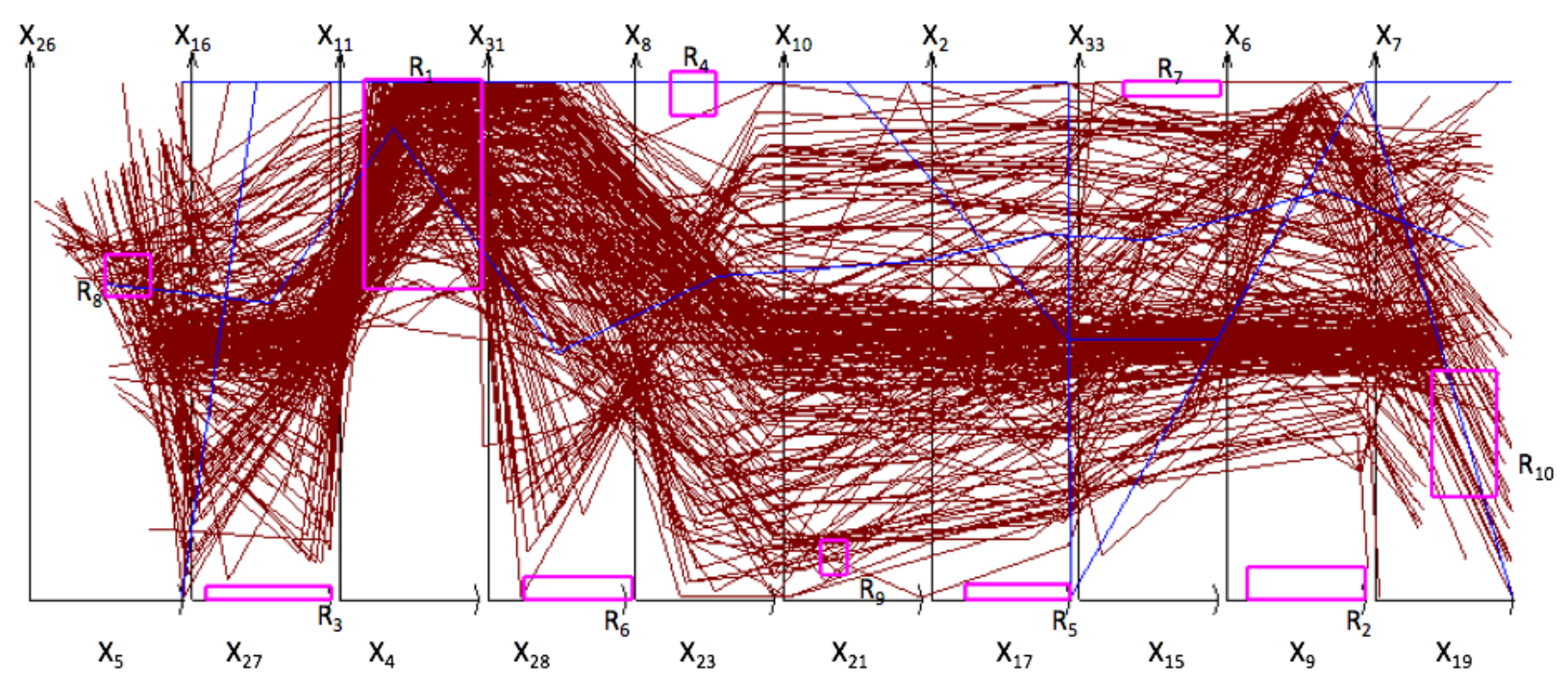

Figure 13: Ionosphere cases in 20-D points covered by the Ionosphere Rule 1. 


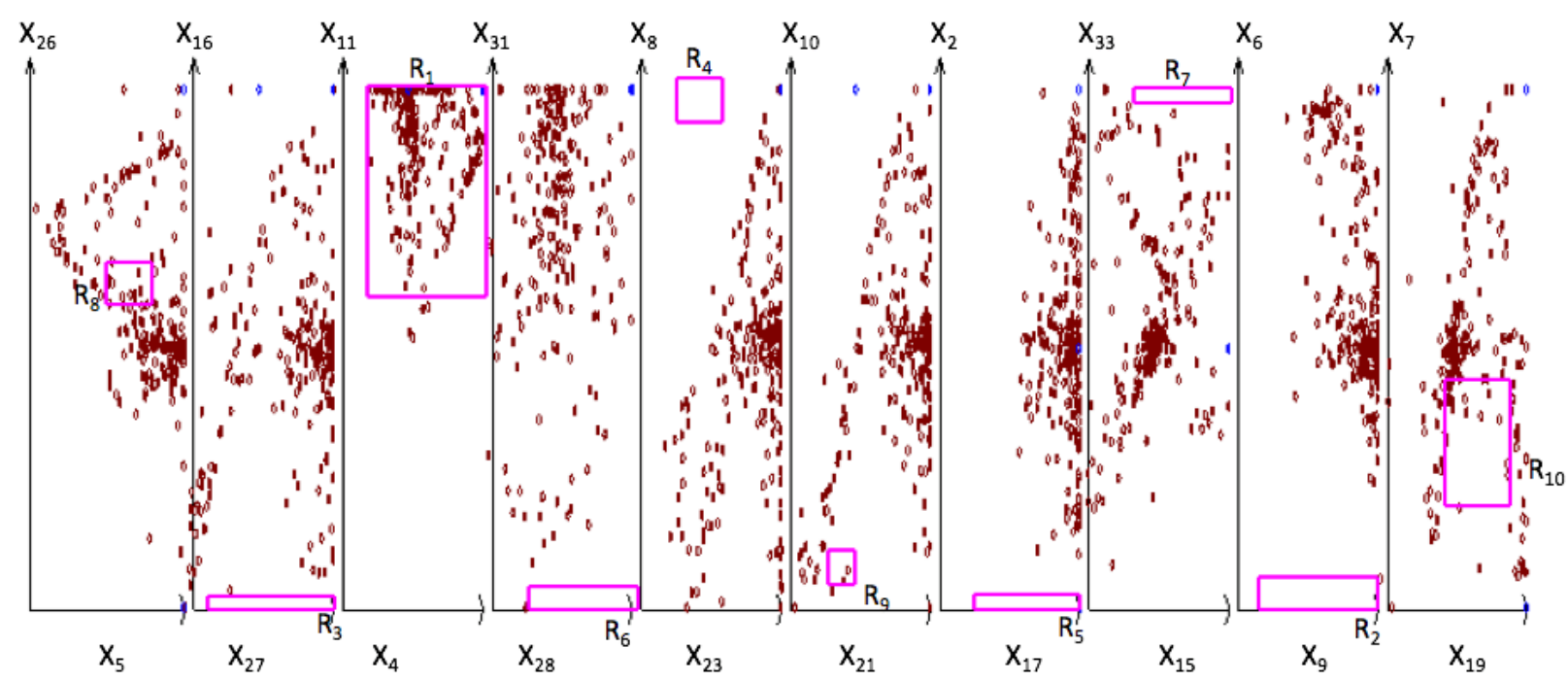

Figure 14: Ionosphere cases in 20-D points covered by Rule 1.

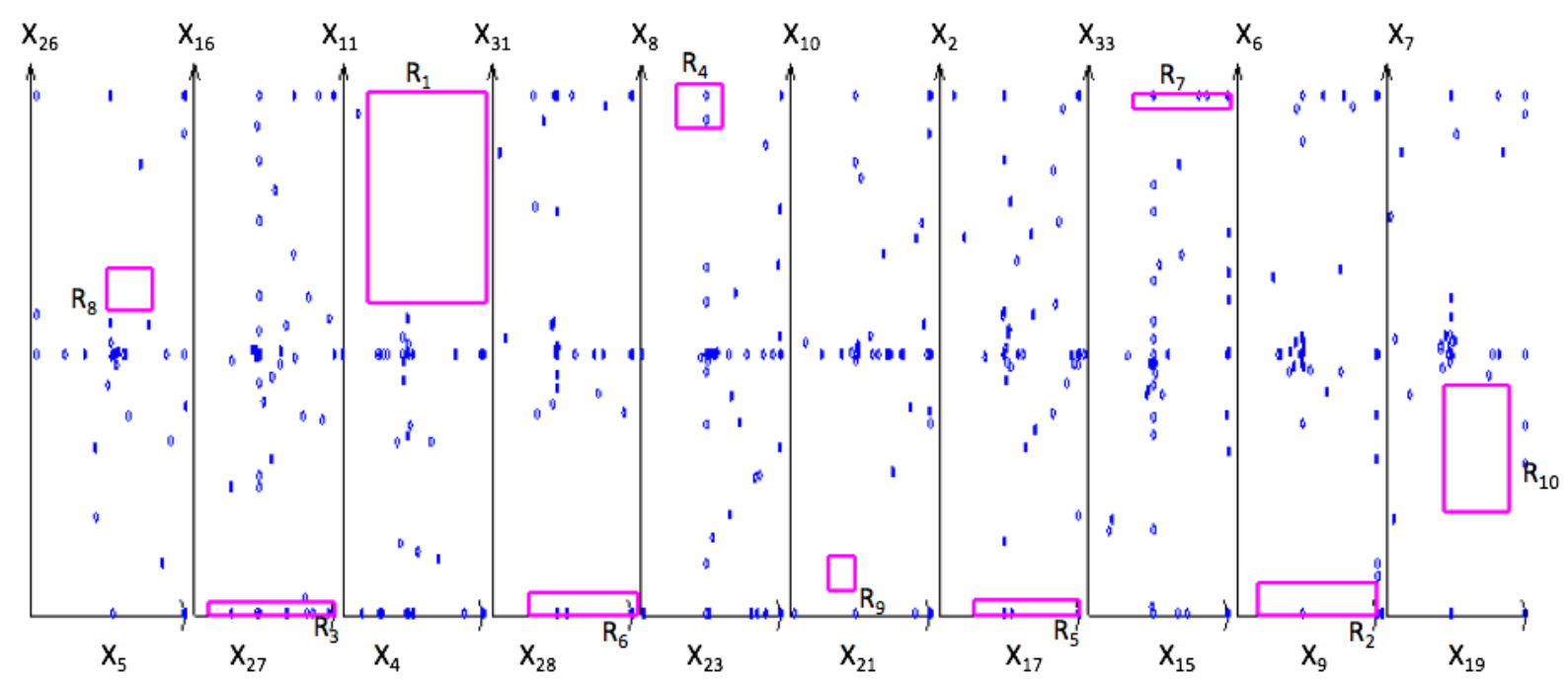

Figure 15: Ionosphere cases in 20-D points not covered by Rule 1.

\section{Experimental Case Study 3}

The computational experiments with the 8-D Abalone data, male and infant cases, from the UCI Machine Learning repository [3] also show the efficiency of the FSP algorithm. The discovered patterns were found by the search in the set of "rectangular" rules (RL1)-(RL8). In 
particular, the FSP algorithm found an efficient sequence of coordinate pairs: $\left(\mathrm{X}_{5}, \mathrm{X}_{6}\right),\left(\mathrm{X}_{1}, \mathrm{X}_{2}\right)$, $\left(\mathrm{X}_{3}, \mathrm{X}_{8}\right),\left(\mathrm{X}_{4}, \mathrm{X}_{7}\right)$. Similarly, to Case Studies 1 and 2, the SPC visualization, with this sequence, reveals the visual classification pattern of with the precision of over $90 \%$ (see Tables 11 and 12) in the 11 random $70 \%: 30 \%$ splits of data into the training and the validation cases. The best precision is $92.06 \%$ on the training data and $96.17 \%$ on the validation data. Fig. 16 shows 2870 Abalone data in the SPCs, as graphs of 8-D points, with the male cases in Red, and the infant cases in Blue. Rectangles used in Abalone Rule 1 defined below are in magenta. Fig.17 and 18 show cases covered this Rule and Fig. 19 shows the remaining cases.

The discovered rules shown in the Tables 11 and 12 belong to the set of rules (RL4). The first Rule in the Table 11, which we denote as the Abalone Rule 1 is:

$$
\begin{aligned}
& \text { If }\left[\left(x_{4}, x_{7}\right) \in R_{1} \vee\left(x_{1}, x_{2}\right) \in\left(R_{2} \vee R_{8}\right) \vee\left(x_{3}, x_{8}\right) \in\left(R_{4} \vee R_{6}\right)\right] \&\left[\left(x_{5}, x_{6}\right) \notin\left(R_{3} \& R_{10} \& R_{11}\right)\right. \\
& \left.\&\left(x_{1}, x_{2}\right) \notin\left(R_{5} \& R_{9}\right) \&\left(x_{3}, x_{8}\right) \notin\left(R_{4} \& R_{7}\right)\right] \text {, then } \mathbf{x} \in \text { class } 1 \text { (Red, Male), }
\end{aligned}
$$

where $R_{1}, R_{2}, R_{3}, R_{4}, R_{5}, R_{6}, R_{7}, R_{8}, R_{9}, R_{10}$, and $R_{11}$ are specific rectangles (see Table 13 ), in respective pairs of the Cartesian coordinates $\left(\mathrm{X}_{4}, \mathrm{X}_{7}\right),\left(\mathrm{X}_{1}, \mathrm{X}_{2}\right),\left(\mathrm{X}_{5}, \mathrm{X}_{6}\right),\left(\mathrm{X}_{3}, \mathrm{X}_{8}\right),\left(\mathrm{X}_{1}, \mathrm{X}_{2}\right),\left(\mathrm{X}_{3}\right.$, $\left.\mathrm{X}_{8}\right),\left(\mathrm{X}_{3}, \mathrm{X}_{8}\right),\left(\mathrm{X}_{1}, \mathrm{X}_{2}\right),\left(\mathrm{X}_{1}, \mathrm{X}_{2}\right),\left(\mathrm{X}_{5}, \mathrm{X}_{6}\right)$, and $\left(\mathrm{X}_{5}, \mathrm{X}_{6}\right)$. The simple Abalone Rule 2, which classifies all the remaining cases (not covered by Rule 1) into class 2 is:

$$
\begin{gathered}
\text { If }\left[\left(x_{4}, x_{7}\right) \in R_{1} \vee\left(x_{1}, x_{2}\right) \in\left(R_{2} \vee R_{8}\right) \vee\left(x_{3}, x_{8}\right) \in\left(R_{4} \vee R_{6}\right)\right] \&\left[\left(x_{5}, x_{6}\right) \notin\left(R_{3} \& R_{10} \& R_{11}\right)\right. \\
\left.\&\left(x_{1}, x_{2}\right) \notin\left(R_{5} \& R_{9}\right) \&\left(x_{3}, x_{8}\right) \notin\left(R_{4} \& R_{7}\right)\right] \\
\text { then } \mathbf{x} \in \text { class } 1 \text { (Red, Male), else } \mathbf{x} \in \text { class } 2 \text { (Blue, Infant) }
\end{gathered}
$$

with accuracy $94.91 \%$ on all cases, $93.33 \%$ and $98.60 \%$ on training and validation data based on the confusion matrixes Table 14. 


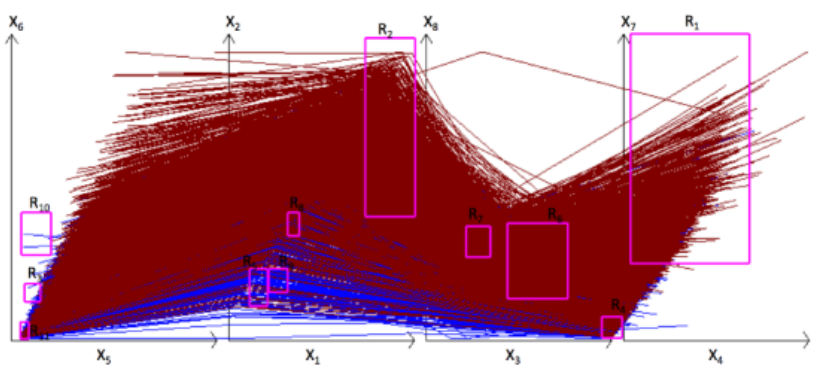

(a) Red cases visualized on the top Blue cases.

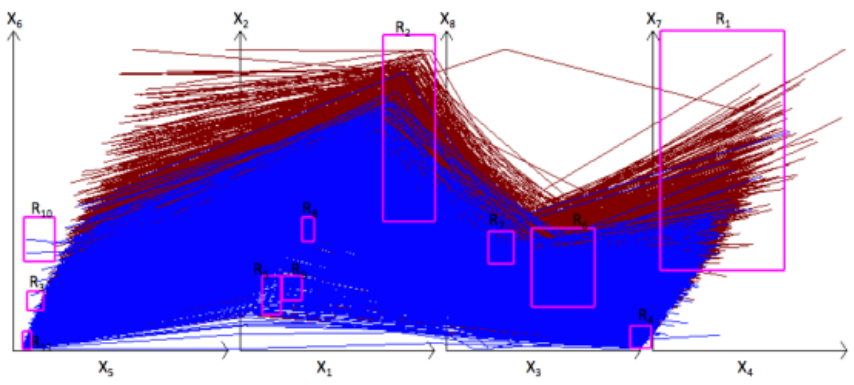

(b) Blue cases visualized on the top the Red cases.

Figure 16: A set of 2870 Abalone data visualized in the SPCs, as graphs of 8-D points, with the male cases in Red, and the infant cases in Blue. Rectangles used in Abalone Rule 1 are in magenta.

Table 11. Number of cases satisfying the if part of Abalone Rule 1 in 11 random 70\%:30\% splits.

\begin{tabular}{|c|c|c|c|c|c|c|}
\hline \multirow{3}{*}{$\begin{array}{c}70 \%: 30 \% \\
\text { random } \\
\text { data splits }\end{array}$} & \multicolumn{6}{|c|}{ Number of cases that satisfy if part of the Rule 1} \\
\hline & & Red class & & & Blue Class & \\
\hline & training & validation & total & training & validation & Total \\
\hline 1 & 1193 & 304 & 1497 & 103 & 12 & 115 \\
\hline 2 & 1034 & 396 & 1430 & 103 & 37 & 140 \\
\hline 3 & 972 & 463 & 1435 & 86 & 46 & 132 \\
\hline 4 & 1015 & 484 & 1499 & 105 & 40 & 145 \\
\hline 5 & 1224 & 272 & 1496 & 126 & 10 & 136 \\
\hline 6 & 1003 & 371 & 1374 & 98 & 28 & 126 \\
\hline 7 & 989 & 402 & 1391 & 92 & 37 & 129 \\
\hline 8 & 1077 & 389 & 1466 & 108 & 22 & 130 \\
\hline 9 & 1146 & 328 & 1474 & 117 & 31 & 148 \\
\hline 10 & 1201 & 276 & 1477 & 121 & 15 & 136 \\
\hline 11 & 996 & 357 & 1353 & 103 & 35 & 138 \\
\hline Average & 1078 & 367 & 1445 & 106 & 29 & 134 \\
\hline
\end{tabular}

Table 12. Precision, recall and coverage of Rule 1 in 11 random 70\%:30\% splits of data.

\begin{tabular}{|c|c|c|c|c|c|c|c|c|}
\hline \multirow{2}{*}{$\begin{array}{c}70 \%: 30 \% \\
\text { random } \\
\text { data splits }\end{array}$} & \multicolumn{2}{|c|}{ Rule precision } & \multicolumn{3}{|c|}{ Rule recall (correct) coverage } & \multirow{2}{*}{$\begin{array}{c}\text { Rule total } \\
\text { coverage of } \\
\text { cases, } \%\end{array}$} & \multicolumn{2}{|c|}{ Rule F measure } \\
\hline & $\begin{array}{c}\text { Training, } \\
\%\end{array}$ & $\begin{array}{c}\text { Validation, } \\
\%\end{array}$ & $\begin{array}{c}\text { Training, } \\
\%\end{array}$ & $\begin{array}{c}\text { Validation, } \\
\%\end{array}$ & $\begin{array}{c}\text { Total, } \\
\%\end{array}$ & & $\begin{array}{c}\text { Validation, } \\
\%\end{array}$ & $\begin{array}{c}\text { Total, } \\
\%\end{array}$ \\
\hline 1 & 92.05 & 96.20 & 41.56 & 10.59 & 52.16 & 56.16 & 0.5727 & 0.1908 \\
\hline 2 & 90.94 & 91.45 & 36.02 & 13.79 & 49.82 & 54.70 & 0.5160 & 0.2397 \\
\hline 3 & 91.87 & 90.96 & 33.86 & 16.13 & 50 & 54.59 & 0.4948 & 0.2740 \\
\hline 4 & 90.62 & 92.36 & 35.36 & 16.86 & 52.22 & 57.28 & 0.5087 & 0.2851 \\
\hline 5 & 90.66 & 96.45 & 42.64 & 9.47 & 52.12 & 56.86 & 0.5800 & 0.1725 \\
\hline 6 & 91.09 & 92.98 & 34.94 & 12.92 & 47.87 & 52.26 & 0.5051 & 0.2269 \\
\hline 7 & 91.48 & 91.57 & 34.45 & 14.00 & 48.46 & 52.96 & 0.5005 & 0.2429 \\
\hline 8 & 90.88 & 94.64 & 37.52 & 13.55 & 51.08 & 55.6 & 0.5311 & 0.2371 \\
\hline 9 & 90.73 & 91.36 & 39.93 & 11.42 & 51.35 & 56.51 & 0.5545 & 0.2030 \\
\hline 10 & 90.84 & 94.84 & 41.84 & 9.616 & 51.46 & 56.2 & 0.5729 & 0.1746 \\
\hline 11 & 90.62 & 91.07 & 34.70 & 12.43 & 47.14 & 51.95 & 0.5018 & 0.2187 \\
\hline Average & 91.07 & 93.06 & 37.53 & 12.80 & 50.33 & 55.01 & 0.5307 & 0.2241 \\
\hline
\end{tabular}




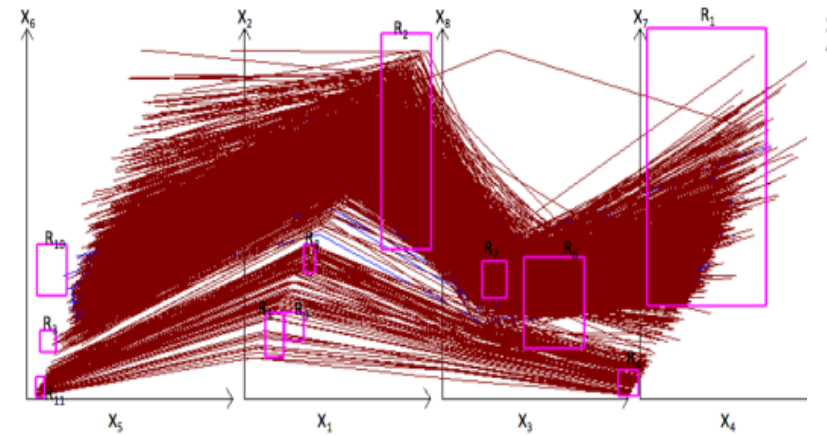

(a) Red cases on the top of the Blue cases.

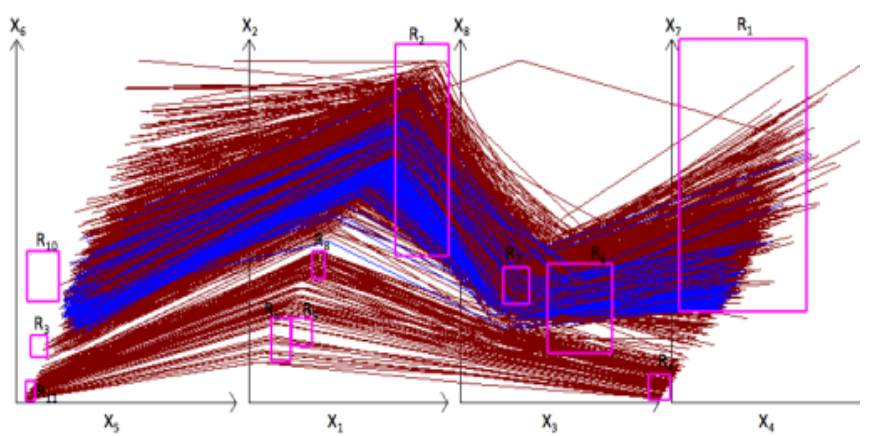

(b) Blue cases on the top of the Red cases.

Figure 17: 8-D Abalone cases covered by Abalone Rule 1.
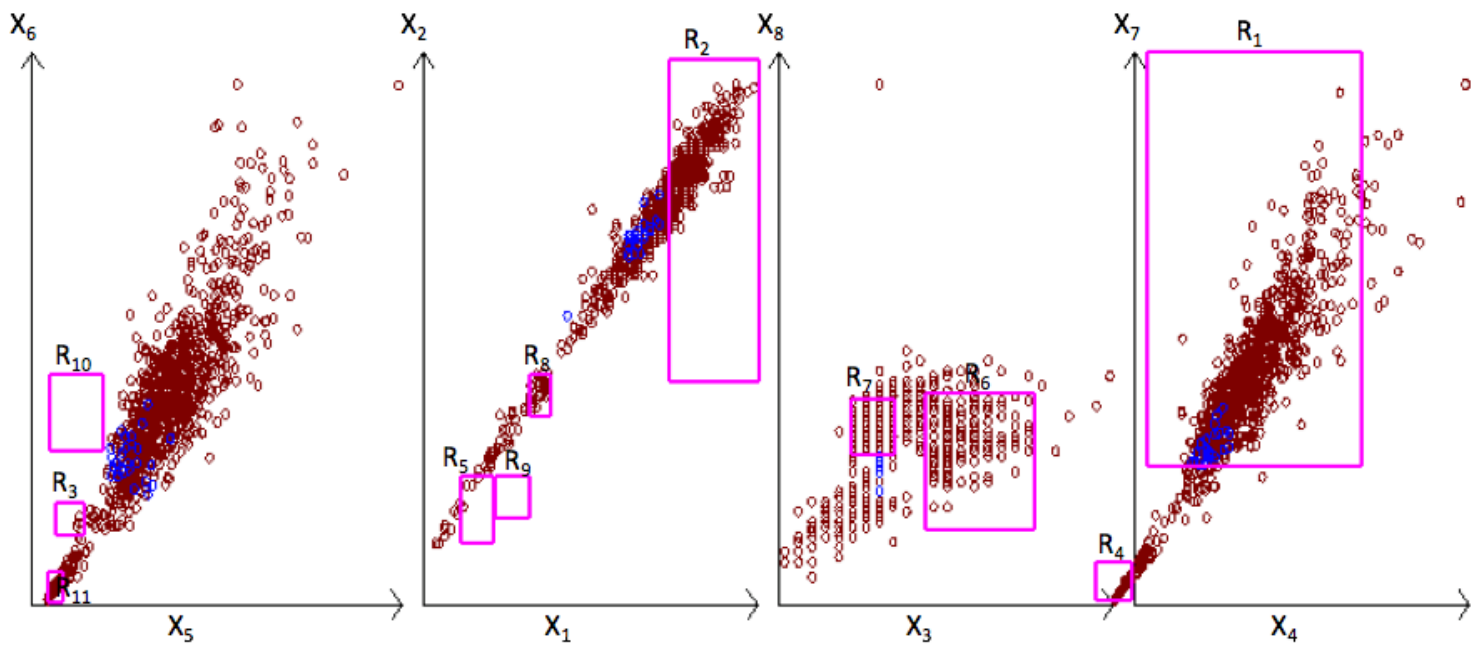

Figure 18: 8-D Abalone cases covered by Rule 1 (only nodes of graphs are shown to decrease occlusion) 

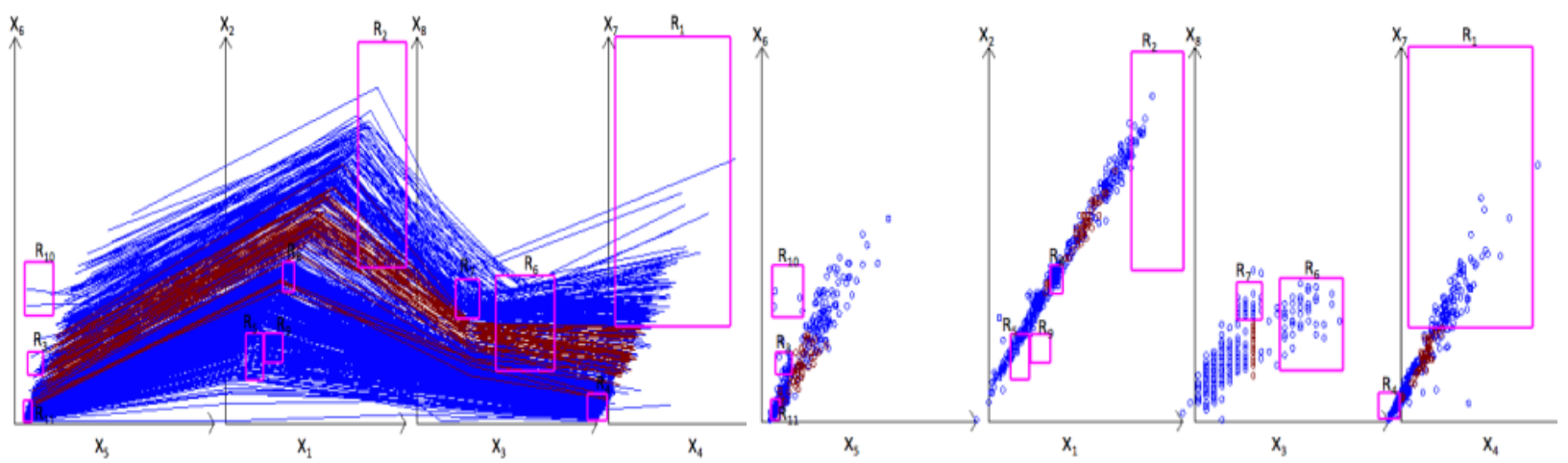

Figure 19: Remaining Abalone cases (cases not covered by Abalone Rule 1).

Table 13. Parameters of rectangles R1-R11.

\begin{tabular}{|c|c|c|c|c|}
\hline \multirow[t]{2}{*}{ Rectangle } & \multicolumn{4}{|c|}{ Parameters } \\
\hline & Left & Right & Bottom & Top \\
\hline$R_{1}$ in $\left(\mathrm{X}_{4}, \mathrm{X}_{7}\right)$ & 0.09068 & 0.6946 & 0.260333 & 1.00 \\
\hline$R_{2}$ in $\left(\mathrm{X}_{1}, \mathrm{X}_{2}\right)$ & 0.7458 & 0.99955 & 0.421667 & 0.99996 \\
\hline$R_{3}$ in $\left(\mathrm{X}_{5}, \mathrm{X}_{6}\right)$ & 0.0209 & 0.1015 & 0.128333 & 0.190667 \\
\hline$R_{4}$ in $\left(\mathrm{X}_{3}, \mathrm{X}_{8}\right)$ & 0.94857 & 1.001 & 0 & 0.077 \\
\hline$R_{5}$ in $\left(\mathrm{X}_{1}, \mathrm{X}_{2}\right)$ & 0.162175 & 0.253525 & 0.242 & 0.113667 \\
\hline$R_{6}$ in $\left(\mathrm{X}_{3}, \mathrm{X}_{8}\right)$ & 0.46645 & 0.77602 & 0.139333 & 0.399667 \\
\hline$R_{7}$ in $\left(\mathrm{X}_{3}, \mathrm{X}_{8}\right)$ & 0.25837 & 0.38017 & 0.282333 & 0.388667 \\
\hline$R_{8}$ in $\left(\mathrm{X}_{1}, \mathrm{X}_{2}\right)$ & 0.355025 & 0.415925 & 0.355667 & 0.436333 \\
\hline$R_{9}$ in $\left(\mathrm{X}_{1}, \mathrm{X}_{2}\right)$ & 0.26 & 0.355025 & 0.161333 & 0.242 \\
\hline$R_{10}$ in $\left(\mathrm{X}_{5}, \mathrm{X}_{6}\right)$ & 0.015075 & 0.167325 & 0.289667 & 0.436333 \\
\hline$R_{11}$ in $\left(\mathrm{X}_{5}, \mathrm{X}_{6}\right)$ & 0.012 & 0.035675 & 0.0 & 0.0586667 \\
\hline
\end{tabular}

Table 14. Confusion matrixes of Abalone Rule 2.

\begin{tabular}{l|lll}
\hline $\begin{array}{l}\text { Actual } \\
\text { class }\end{array}$ & \multicolumn{4}{|l}{$\begin{array}{l}\text { Confusion matrix on } \\
\text { all 2870 cases }\end{array}$} \\
& \multicolumn{4}{|l}{$\begin{array}{l}\text { Predicted class } \\
\text { Red }\end{array}$} & Blue & Total \\
Red & 1497 & 115 & 1612 \\
Blue & 31 & 1227 & 1258 \\
Total & 1528 & 1342 & 2870 \\
\hline
\end{tabular}

\begin{tabular}{|c|c|c|}
\hline \multicolumn{3}{|c|}{$\begin{array}{l}\text { Confusion matrix on trai- } \\
\text { ning cases }(2009,70 \%)\end{array}$} \\
\hline \multicolumn{3}{|c|}{ Predicted class } \\
\hline Red & Blue & Total \\
\hline 1193 & 103 & 1296 \\
\hline 31 & 682 & 713 \\
\hline 1224 & 785 & 2009 \\
\hline
\end{tabular}

\begin{tabular}{|c|c|c|}
\hline \multirow{2}{*}{\multicolumn{3}{|c|}{$\begin{array}{l}\text { Confusion matrix on } \\
\text { validation cases }(861,30 \%) \\
\text { Predicted class }\end{array}$}} \\
\hline & & \\
\hline Red & Blue & Total \\
\hline 304 & 12 & 316 \\
\hline 0 & 545 & 545 \\
\hline 304 & 557 & 861 \\
\hline
\end{tabular}

\section{Experimental Case Study 4}

In order to test the FSP method on high dimensional data, a set of Modified National Institute of Standards and Technology (MNIST) Database of handwritten digits [14] has being used. 
These computational experiments conducted with digits 0 and 1 represented as the 484-D points (cases) show the efficiency of the FSP algorithm. The discovered patterns were found by the search in the set of "rectangular" rules (RL1)-(RL8). The training data contain 7030 and 7781. The discovered Rule1 has a form of a Rule 4 (see page 11). It contains 132 rectangles that are spread around 78242 paired coordinates with average of 1.69 rectangles per pair. In contrast with case studies $1-3$, in this case study we did not conduct pair permutation and used the original order of pairs: $\left(\mathrm{X}_{1}, \mathrm{X}_{2}\right),\left(\mathrm{X}_{3}, \mathrm{X}_{4}\right), \ldots,\left(\mathrm{X}_{\mathrm{n}-1}, \mathrm{X}_{\mathrm{n}}\right)$ due to multiplicity of pairs.

This multiplicity also creates a challenge to visualize all 484 dimensions in SPC that are used by FSP algorithm. This reveals one of the limitations of FSP algorithm based on SPC for the direct visualization of discrimination rules. Therefore, below we propose a generalized visualization of a discrimination rules for such high-dimensional data (images). The idea of a new visualization is to "overlay" the discrimination Rule1 on the input images of digits.

The steps of the new algorithm that we denote as R2I (Rectangles In Image) below:

(a) Compute the average image $\mathrm{M}(0)$ for digit 0 by averaging all respective pixels of the training data of this digit:

$$
\mathrm{M}(0)=\left\{m(i, j): \mathrm{m}(i, j)=\text { average }_{k \in K}(\operatorname{Tr}(0, k, i, j)\}\right.
$$

where $K$ is the number of digits 0 in the training data and $\operatorname{Tr}(0, k, i, j)$ is the intensity of the pixel $(i, j)$ of the k-th image of digit 0 . See figure 20 .

(b) Compute the average image $\mathrm{M}(1)$ for digit 1 by averaging all respective pixels in the same way as in (a). See figure 20. 
(c) Compute the average image $\mathrm{T}$ for digit 0 and 1 by averaging $\mathrm{M}(0)$ and $\mathrm{M}(1)$. figure 22 .

(d) Find location of the pairs of pixels in the image 21 for digit 0 that have been used in rectangles in the discovered Rule1. For instance, let pixels 71 and 72 form a pair of coordinates $\left(\mathrm{X}_{71}, \mathrm{X}_{72}\right)$ and the rectangle $\mathrm{R} 1$ from Rule1 is discovered in these coordinates.

(e) Show pixels 71 and 72 in black. Do this for all rectangles involved in the Rule 1. The result is in figure 21 and figure 22.

(f) Conduct steps (d)-(e) for digit 1. The result is in Figure 21 and figure 22.

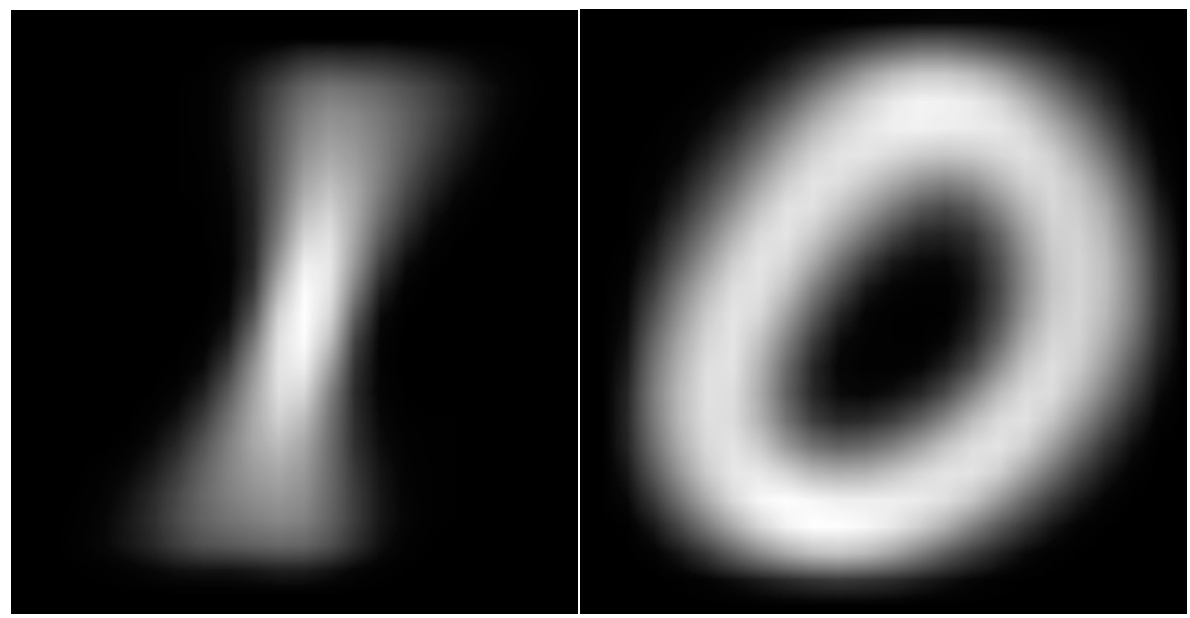

Figure 20: Sum and average digits 0 and 1 in gray scale

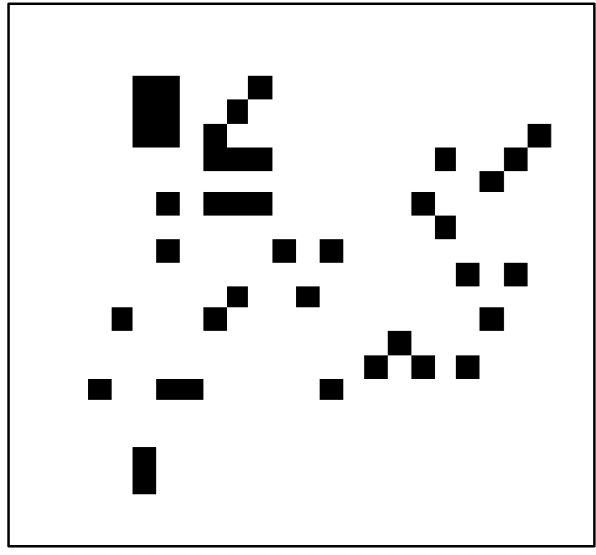

Figure 21: SPC pairs presented as black dots in 22x22 empty image for 1 and 0 . 


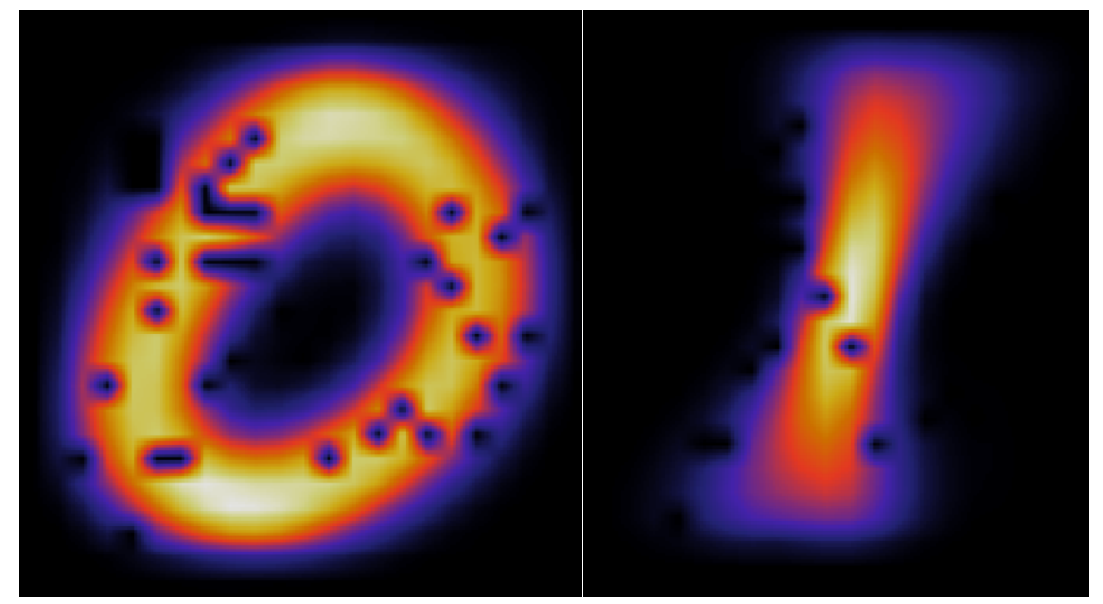

Figure 22: SPC pairs presented as dark dots in the average of sum image of 1 and 0.

The result has a precision over $90 \%$ in random 70\%:30\% splits of data into the training and the validation date. The best precision obtained is $96.21 \%$ on the training data and $98.03 \%$ on the validation data. Fig. 21 represents the SPC pairs locations as a dark dot in an empty $22 \times 22$ image. Fig. 22 shows 2115 MINST data in the average of sums for number 0 and 1 as an image of $22 \times 22$ pixels (484 dimensions). The dark dots represent pairs that been covered by the rules.

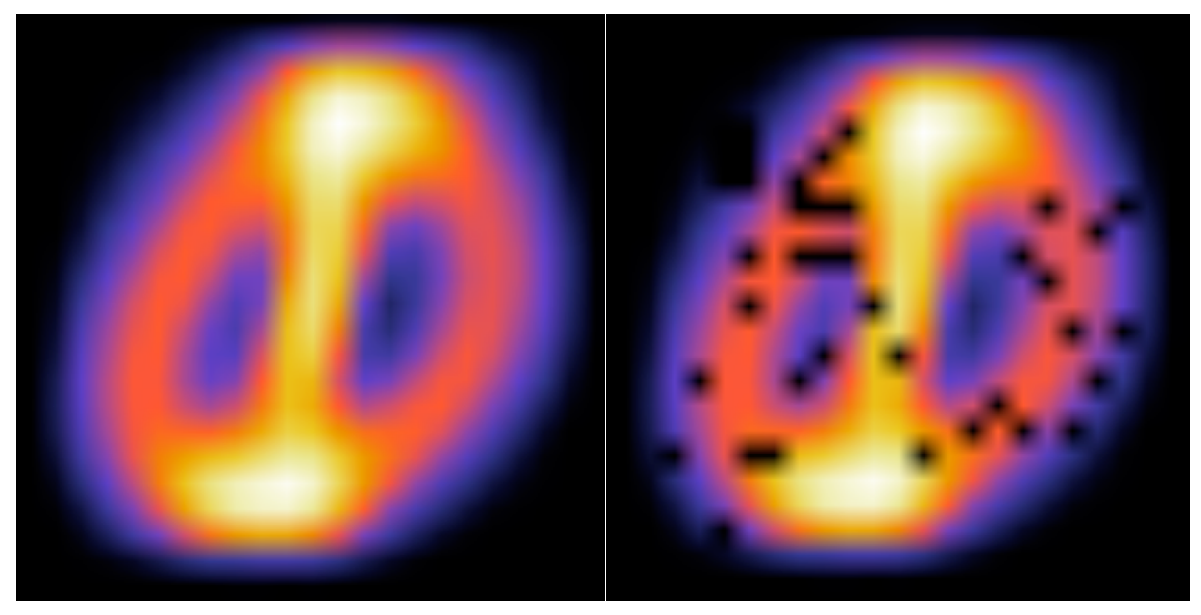

Figure 23: SPC pairs presented as dark dots in the average of sum image of 1 and 0. 


\section{CHAPTER IV}

\section{CONCLUSION}

\section{Comparison with Published Results}

Case study 1: the best accuracy reported for the Wisconsin Breast Cancer (WBC) dataset for the SVM in [6] is $96.995 \%$ with the 10 -fold cross-validation tests. Other results are $96.84 \%$ [7] and $96.99 \%$ [8] for the SVM, and 97.28\% [6] by combing SVM, C4.5 decision tree, naïve Bayesian classifier, and the k-Nearest Neighbors algorithms.

These models classify all the cases, while many of our rules refuse to classify some of the cases. Our WBC Rule 2 classify all cases, but with lower accuracy 93.60\%. Our better Rule that combines WBC rules 1 and 3 has precision 95.18\%. While in general, accuracy and precision are different, here the combination of rules 1 and 3 cover almost all cases $(99.42 \%$, only 4 cases are refused). The precision for such high coverage is almost identical to accuracy. Thus, it is quite close to the published results, but slightly lower. However, in contrast with SVM, it is visual, has clear interpretation and explainable to a domain expert which is very important in domains with high cost of errors where the explanation of the model is mandatory.

Case study 2: for Ionosphere dataset, the highest accuracy reported by [9] is $98 \%$ on training and $93 \%$ on validation data using the multilayer perceptron. Other results are $94.87 \%$ by using C4.5 algorithm and 94.59\% using Rule Induction RIAC algorithm [10], 97.33\% by SVM with Particle Swarm Optimization and 10-fold cross-validation [11].

These models classify all the objects, while many of our rules refuse to classify some of the cases. In contrast, our Ionosphere Rule 2 classify all cases. For this Rule, precision is identical to accuracy that is $98.78 \%$ on training data and $100 \%$ on validation data. Thus, our results are slightly higher, than those for the published classification models. 
Case study 3: The highest accuracy reported in [12] for Abalone dataset using SVM is $99.26 \%$ with 5-fold Cross-validation for all three classes. Another result is $97.80 \%$ accuracy using a case base reasoning method [13]. Our result, that are within $[93.33,98.60] \%$ interval, are quite close to these published results. Unlike [12], we use a more challenging approach for classification 70:30 split, than the 5-fold that is 80:20 split.

The goal of [15] was to find large empty rectangles or boxes in $1 \mathrm{D}, 2 \mathrm{D}, 3 \mathrm{D}, 4 \mathrm{D}$, and 5D spaces. This goal differs from our goal of finding 2-D rectangles filled by points of a single class or dominated by that class. This we have a "reverse" task. Also, the focus of [15] is designing a new computationally efficient algorithm to find holes in high dimensional data that runs in polynomial time. This is different from FSP algorithm too. Unlike the empty rectangles in [15], FSP algorithm use the rectangles in 2D to classify data. Also, FSP try to find rectangle that contains the points that belong to specific class and reduce the error by finding another rectangle that classify the wrong cases in the first rectangle. The potential use in [15] for strengthening FSP using algorithm from [15] to search most non-empty rectangles in 2-D or remove most empty before searching for non-empty. For non-empty rectangles it is likely that algorithm from [15] must be modified.

\section{Conclusion}

The FSP Rule 2 for Wisconsin Breast Cancer (WBC), Ionosphere and Abalone are visual, interpretable, and explainable to a domain expert, which is critical, in many domains with mandatory model explanation. This comparison shows that the proposed FSP algorithm, with the SPC visualization, produced the results comparable with the other major machine learning algorithms, in the accuracy and precision. The FSP algorithm has the following significant 
advantages: it is (i) visual with minimal occlusion, (ii), interactive, (iii) understandable by the user, and (iv) simpler than many machine-learning algorithms.

Future study would focus on using more interpretable rules for discovery by the FSP algorithm along with the other General Line Coordinates, beyond SPC. Also, to optimize the search time, by discard the highly correlated coordinates in random generation of pairs. That reduces run time between 5 to 30 percent in some cases. Another way to optimize the search of rectangles is to use one of the evolutionary algorithms instead of purely random ones to find the best possible sequence and pairs. The directed search will increase the chance of reducing the time and improving the accuracy. 


\section{REFERENCES}

1. Kovalerchuk B., Gharawi A., Decreasing Occlusion and Increasing Explanation in Interactive Visual Knowledge Discovery, In: Human Interface and the Management of Information. Interaction, Visualization, and Analytics, Lecture Notes in Computer Science series, Vol. 10904, 2018, Springer

2. Kovalerchuk, B., Grishin, V. Adjustable general line coordinates for visual knowledge discovery in n-D data. Inf. Vis. 2017, doi:10.1177/1473871617715860.

3. Kovalerchuk B. Visual Knowledge Discovery and Machine Learning, 2018, Springer.

4. Lichman, M. UCI Machine Learning Repository, http://archive.ics.uci.edu/ml, 2013.

5. Wilinski, A., Kovalerchuk, B. Visual knowledge discovery and machine learning for investment strategy. Cognitive Systems Research, v. 44, 2017, pp. 100-114.

6. Salama, G.I.,Abdelhalim, M., Zeid, M.A. Breast cancer diagnosis on three different datasets using multi-classifiers. Breast Cancer (WDBC) 2012, 32, 2.

7. Aruna, S., Rajagopalan, D.S., Nandakishore, L.V. Knowledge based analysis of various statistical tools in detecting breast cancer. Comput. Sci. Inf. Technol. 2011, 2, 37-45.

8. Christobel, A., Sivaprakasam, Y. An empirical comparison of data mining classification methods. Int. J. Comput. Inf. Syst. 2011, 3, 24-28.

9. Duch W,Kordos M.Multilayer perceptron with numerical gradient.ICANN2003,106-109.

10.Hamilton HJ, Shan N., Cercone N., RIAC: a Rule induction algorithm based on approximate classification. Computer Science Department, University of Regina; 1996.

11.Tu, C.-J., Chuang, L, Y., Yang, C.H. Feature selection Using PSO-SVM. IAENG Int. J. Comput. Sci. 33(1), 111-116,2007

12.Sain H., Purnami SW., Combine sampling support vector machine for imbalanced data 
classification. Procedia Computer Science. 2015,1;72:59-66.

13.Smiti A., Elouedi Z. Maintaining Case Based Reasoning Systems Based on Soft Competence Model. In Intern. Confer. on Hybrid Artificial Intelligence Systems 2014,666-677. Springer. 14.Y. LeCun and C. Cortes, "MNIST handwritten digit database, http://yann.lecun.com/exdb/mnist/, 2010. 


\section{APPENDIXS \\ APPENDIX A \\ WISCONSIN BREAST CANCER (WBC)}

Wisconsin Breast Cancer (WBC) dataset from the UCI machine learning repository [3]. WBC dataset contains 699 instances with 11 attributes. The patient ID was removed from the first dimension. Also, each instance that contains a missing value was removed in the preprocessing phase. That produces 688 records with 448 of benign and 240 with malignant cases. In addition, the dataset was normalized between 0 and 1 .

\section{APPENDIX B}

\section{IONOSPHERE DATASET}

Ionosphere dataset is from the UCI machine learning repository [3]. This dataset contains 351 instances with 35 attributes where $35^{\text {th }}$ dimension represents the class label of good cases and bad cases. In the preprocessing step, the second dimension was removed because it only contains zeros resulting 34-D. Also, the dataset was rescaled between 0 and 1 .

\section{APPENDIX C}

\section{ABALONE DATASET}

Abalone dataset is a dataset from the UCI machine learning repository [3]. This dataset contains 4177 instances with eight attributes for predicting the age of Abalone from physical measurements [3]. The $8^{\text {th }}$ attribute represents the classes label. In the preprocessing step, cases of the female were removed resulting 1612 male and 1258 infant cases. 


\section{APPENDIX D}

\section{MNIST-DATASET}

In order to test the FSP method on high dimensional data, the Modified National Institute of Standards and Technology (MNIST) [14] was used. MNIST Database originally consists of images for digits from 0 to 9 with $28 \times 28$ pixels ( 784 dimensions) for each image. The digits 0 and 1 in the validation dataset was used after removing the padding. The preprocessed images contain a total of 2115,1135 images of digit 1 and 981 of digit 0 with $22 \times 22$ pixels (484 dimensions) each. 